\title{
Inductive Reasoning in Social Choice Theory
}

\author{
Fernando Tohméa $^{a, b}$ - Federico Fioravanti ${ }^{a, b}$ - Marcelo Auday ${ }^{a}$ \\ ${ }^{a}$ Universidad Nacional del Sur \\ ${ }^{b}$ INMABB (CONICET - UNS) \\ Av. Alem 1253, (8000) Bahía Blanca, Argentina \\ e-mail: ftohme@criba.edu.ar, federico.fioravanti9@gmail.com, \\ marceloauday@gmail.com
}

\begin{abstract}
The usual procedure in the theory of social choice consists in postulating some desirable properties which an aggregation procedure should verify and derive from them the features of a corresponding social choice function and the outcomes that arise at each possible profile of preferences. In this paper we invert this line of reasoning and try to infer, up from what we call social situations (each one consisting of a profile and the associated social ordering) the criteria verified in the implicit aggregation procedure. This inference process, which extracts intensional from extensional information can be seen as an exercise in "qualitative statistics".
\end{abstract}

Keywords: Induction; Aggregation; Social Situations.

\section{Introduction}

Social outcomes arise from rather complicated decision-making arrangements among individuals and organizations. The study of these processes of aggregation of individual opinions yielding collective decisions gained momentum in the late 1940s. K. Arrow proved that a very simple set of conditions on the aggregation process, intended to represent the features of democratic systems, were impossible to be fulfilled simultaneously (Arrow 1951). Since then a great number of conditions both limiting and expanding the possibilities of aggregation have been found. 
The literature on the subject starts by postulating conditions for the aggregation processes and then deriving their properties, particularly those related to its implicit "power structure" (i.e. whether it is dictatorial, oligarchic, etc.). Here instead we will start with the class of possible inputs/outputs of those processes. Then we will look for a partition of this class according to the equivalence among decision-making structures and seek to determine the conditions that characterize each equivalence class. This means that instead of deriving results from the conditions on the aggregation procedure, we will characterize those conditions based on its inputs/outputs.

This procedure, which consists in deriving an intensional characterization up from an extensional one is typical in Statistics. This is why we think of this paper as an exercise in qualitative statistics. As we will see, there is no complete equivalence between intensional and extensional characterizations of social choice functions. Even so, some partial information can be drawn by means of this procedure.

Any induction process depends on a previous relevance criterion. That is, on a way of deciding which features should be taken into account and which not. In the specific exercise we develop here, we focus on the idea that names of alternatives are irrelevant. In other words, we assume the well-known condition of neutrality. Notice that we use it here in a radically different way than in the literature, where it is a property predicated on the aggregation procedure. Here is an external condition we impose as analysts to identify different profiles of preferences and social outcomes, classifying them as being equivalent. ${ }^{1}$

A crucial step in our induction process is the definition of an equivalence relation among input/output pairs of aggregation procedures, which we call social situations, based on their decision structures. More precisely, the equivalence is obtained by identifying (under the assumption of neutrality) the decisive sets of those social situations.

Our approach is not without shortcomings, most notably the inability to generate analytical descriptions of aggregation procedures. We are only able to give a description of the decision structure supported by them and moreover translate that into a formal theoretical language. Other relevant properties of aggregation procedures may get lost by the application of the criteria of neutrality and decision-equivalence to classify situations. Again,

\footnotetext{
${ }^{1} \mathrm{~A}$ related distinction, between criteria and axioms, is developed by Campbell and Kelly (1997).
} 
this is an inevitable result of applying an inductive inference, compensated by the possibility of detecting patterns in classes of social situations. In particular, our approach allows detecting all the properties satisfied by voting rules (Austen-Smith \& Banks, 1998).

The plan of the paper is as follows. Section 2 introduces the general ideas of this paper and discusses the intended application of our results. In section 3 we present the partition of social choice situations in terms of permutations of the names of both agents and situations. In section 4 we derive the aggregation functions that correspond to the equivalence classes found in the previous section. In section 5 we relate the aggregation functions with social choice criteria (verified by the elements in the corresponding equivalence class) and extract some properties of the aggregation function. Finally, section 6 concludes.

\section{Elicitation of Axioms}

The study of resource allocation mechanisms ${ }^{2}$ provides a general framework of analysis in which we can state the main objectives of this paper. Unlike that literature, we do not start with a description of the properties of the process that leads from the preferences and endowments of the agents to a social outcome. ${ }^{3}$ We start instead from the inputs and outputs of such process and try to infer its properties. Of course, it is not enough to consider only a single instance and thus we envision a class of environments $\mathcal{E}$ and a family of (social) alternatives $\mathbf{Z}$. Each environment is an exhaustive description of the relevant characteristics of the agents in the society. That is, it provides all the information about the inputs for a social correspondence $\mathbf{f}: \mathcal{E} \rightarrow \mathbf{Z}$ that yields which alternatives are chosen at each environment.

The traditional problem in this setting is:

- Aggregation: given a family of axioms $\mathcal{A}$ representing some desired properties, prove the existence and obtain the characterization of $\mathbf{f}$ such that $\mathcal{A}$ is satisfied.

\footnotetext{
${ }^{2}$ See Hurwicz (1960), Mount \& Reiter (1974), Reiter (1977), Reichelstein \& Reiter (1988) and Hurwicz \& Reiter (2001), among others.

${ }^{3} \mathrm{~A}$ textbook example involves an environment consisting of the preferences of the agents about a public good (for instance whether to build or not a bridge, or to install or not a park in the neighborhood, etc.) and their willingness to pay for it. The social outcome will be the decision of allocate or not resources to the public good and how much to extract from each agent to cover the costs.
} 
We intend to solve the converse problem. Given any $E \subseteq \mathcal{E}$ and $\mathbf{f}=$ $\{\langle e, z\rangle: e \in E, z \in \mathbf{Z}\} \subseteq \mathcal{E} \times \mathbf{Z}$ we want to find the properties satisfied by this social correspondence. Notice that $\mathbf{f}$ may only have an extensional characterization, lacking a full-fledged analytic expression. Then, only a partial list of its properties can be enumerated.

Determining the properties of $\mathbf{f}$ up from $\{\langle e, z\rangle: e \in E, z \in \mathbf{Z}\}$ involves an inductive inference, whose study is the goal of this paper. Since for the general setting given above the problem may be extremely complicated, we restrict it to a framework in which each $e \in E$ is just a profile of individual orderings over a set $S$ of alternatives while $\mathbf{Z}$ are (social) orderings of $S$. The elements in this restricted family of pairs $\{\langle e, z\rangle: e \in E, z \in \mathbf{Z}\}$ are called social situations. In this case, $\mathbf{f}$ is a social welfare function that assigns a social ordering to a profile of individual preferences. Both the inputs and outputs of the process represented by $\mathbf{f}$ are the components of social situations.

These situations differ in either the individual preference orderings or in the social orderings. Even so, if a certain form of neutrality is postulated (indicating that the names of alternatives do not matter, up to permutations), equivalences among the social situations can be found. We call this kind of relation a decision-theoretic equivalence, denoted $\simeq_{\mathcal{D}}$. It defines a partition of the space of situations. Each equivalence class supports a function, which we call prime. The aggregation (set-theoretical union) of equivalence classes yields a new partition, in which each class corresponds to a social choice function. The latter can be factored in terms of its underlying prime functions.

This homomorphic relation between partitions and classes of functions indicates that in order to extract the features verified by $\mathbf{f}$ it suffices to look at its corresponding equivalence class. But the trick here is to specify adequately the information corresponding to the elements of that class. Coalitions of individuals are labeled with some data: the pairs of alternatives on which they are decisive and the permutations of names of alternatives that leave their decisiveness invariant.

The method presented here yields the richest results when the class of decision sets matches one in a "library" of well-known ones. But even if there is no previous known structure to which associate the decision sets obtained by the procedure, this information is useful, since it describes the behavior of the implicit aggregation function (that is now described by its decision sets).

The next sections will show the stepwise development of this method of 
extraction of the implicit information about $\mathbf{f}$.

\section{Partitions of the Space of Social Choice Situa- tions}

We will define a social choice situation with $n$ individuals over a countable set of alternatives $S(|S| \geq 3)$ as $\mathcal{S}=\left\langle S, R_{1}, \ldots, R_{n} ; \bar{R}\right\rangle$, where each $R_{i} \subseteq S^{n}$ is agent $i$ 's preference ordering and $\bar{R} \subseteq S^{n}$ is the social ordering on $S$. We do not place any restriction on the social order. In particular, we do not require $\bar{R}$ (unlike the individual preferences) to be a complete ordering.

We denote $R_{i}(s, t)$ when agent $i$ prefers (not necessarily in a strict way) alternative $t$ to alternative $s$. We restrict our attention to the situations in which each individual ordering, $R_{i} \in\left\{R_{1}, \ldots, R_{n}\right\}$, is:

- reflexive: $R_{i}(s, s)$ for each $s \in S$.

- complete: $R_{i}(s, t)$ or $R_{i}(t, s)$ for every $s, t \in S$.

- transitive: if $R_{i}(s, t)$ and $R_{i}(t, u)$ then $R_{i}(s, u)$ for $s, t, u \in S$.

These conditions, that define $R_{i}$ as a weak order, are usually seen as representing the rationality (consistency) of a decision-maker. The class of situations described so far is denoted $\widehat{\mathcal{S}}$.

We will assume that the expression social choice situation applies only to those in which the profiles of preferences verify these conditions. As we wish to classify situations and then extract the information of the conditions satisfied by the social choice situations, we should place ourselves in the position of an outsider observer who has to decide which features must be deemed relevant and which are just accessory ones.

The first point we make is that if we are trying to find a commonality between different social choice situations it is convenient to think that the names of alternatives are irrelevant. The reason for this choice is that if we try to detect, say, the presence of the majority or the plurality rule, it does not matter which are the actual alternatives: those rules act in the same way independently of the names of the elements in $S .{ }^{4}$ In our case $S$ is fixed, and so, to apply this criterion, we assume that the family of admissible

\footnotetext{
${ }^{4}$ Notice that we could also apply anonymity, i.e. independence of the names of the agents. But then, for instance, all dictatorial rules would become identified, critically reducing the number of aggregation procedures that our inductive method is able to characterize.
} 
automorphisms ("name changes") over $S$ constitutes a group. For this it is enough to take $\mathcal{G}_{S}=\{\gamma: S \rightarrow S\}$ to be the set of all possible permutations of elements in $S$.

Keeping in mind that we treat the names of social alternatives as if they were irrelevant, we are interested in identifying different social situations through an equivalence relation. The basic notion on which such an equivalence can be defined is that of decisive sets. As defined by Arrow, a decisive set for an alternative $s$ over another $t$ is the family of agents such that, if each of them prefers $s$ to $t$, then in the social order $s$ must be above $t$. That is, a decisive set represents the individuals that, if they agree on the preference over two alternatives, they can impose this preference on the rest of the society.

We will then consider that two social situations are equivalent if their decisive sets are the same under a renaming of the alternatives. In this way, we capture the idea that the agents behave analogously in both social situations and thus the corresponding social choices are the same up to a renaming of the alternatives. Formally, we define a binary relation $\widehat{\mathcal{S}}$, $\simeq_{\mathcal{D}\left(\mathcal{G}_{S}\right)}$, which we call decision-equivalence:

Definition 1 Two social choice situations, $\mathcal{S}=\left\langle S, R_{1}, \ldots, R_{n} ; \bar{R}\right\rangle$ and $\mathcal{S}^{\prime}=$ $\left\langle S, R_{1}^{\prime}, \ldots, R_{n}^{\prime} ; \bar{R}^{\prime}\right\rangle$ are decision-equivalent, $\mathcal{S} \simeq_{\mathcal{D}\left(\mathcal{G}_{S}\right)} \mathcal{S}^{\prime}$, if and only if there exist a permutation $\gamma \in \mathcal{G}_{S}$ such that for each pair $s, t \in S$ we have that

$$
\begin{gathered}
D^{\mathcal{S}}(\{s, t\})=\left\{i: R_{i}(s, t) \text { iff } \bar{R}(s, t)\right\}= \\
=\left\{i: R_{i}^{\prime}(\gamma(s), \gamma(t)) \text { iff } \bar{R}^{\prime}(\gamma(s), \gamma(t))\right\}=D^{\mathcal{S}^{\prime}}(\{\gamma(s), \gamma(t)\})
\end{gathered}
$$

If $\bar{R}(s, t)$ is undefined, we state by convention that $D^{\mathcal{S}}(\{s, t\})=\emptyset$.

In words: two social choice situations are decision-equivalent if and only if there exist a renaming of the alternatives (an automorphism in $\mathcal{G}_{S}$ ) that makes their decisive sets coincide. Informally, this amounts to saying that two situations are equivalent if their implicit "power structure" (i.e. the coalitions of agents that impose their will over the rest) is the same, except for the renaming of alternatives. The intuition is that both situations result from applying the same aggregation procedure, which is independent of the names of the alternatives. 
Example 1 Consider two situations in which three agents (1,2 and 3$)$ have to decide: ${ }^{5}$

$$
\mathcal{S}=\left\langle\{a, b, c\},\{a \prec b \prec c\}_{1},\{b \prec c \prec a\}_{2},\{a \prec b \prec c\}_{3} ;\{\{b \prec c\},\{a\}\}\right\rangle
$$

and

$$
\mathcal{S}^{\prime}=\left\langle\{a, b, c\},\{a \prec c \prec b\}_{1},\{a \prec b \prec c\}_{2},\{b \prec a \prec c\}_{3} ;\{\{a \prec c\},\{b\}\}\right\rangle .
$$

In both situations, the agents order linearly the three alternatives $a, b, c$ and the social outcome is similar: one alternative is preferred to another, while the remaining one does not bear any relation with the other two alternatives. We have that

$$
D^{\mathcal{S}}(\{b, c\})=\{1,2,3\}=D^{\mathcal{S}^{\prime}}(\{a, c\})
$$

while, since one alternative is not related in the social orderings to the other two, we have that

$$
D^{\mathcal{S}}(\{b, a\})=D^{\mathcal{S}}(\{a, c\})=\emptyset=D^{\mathcal{S}^{\prime}}(\{b, a\})=D^{\mathcal{S}^{\prime}}(\{b, c\})
$$

That is, $\mathcal{S} \simeq_{\mathcal{D}} \mathcal{S}^{\prime}$ under $(12)$.

We can see here that thanks to our equivalence relation, we can both simplify and enrich the information we are working with.

We have that: ${ }^{6}$

Proposition 1 The class $\widehat{\mathcal{S}}$ of social choice situations is partitioned by $\simeq_{\mathcal{D}\left(\mathcal{G}_{S}\right)}$.

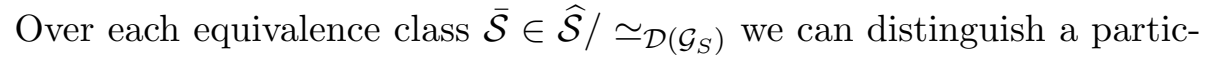
ular class of decisive sets which will allow us to identify the class:

Definition 2 For $\overline{\mathcal{S}} \in \widehat{\mathcal{S}} / \simeq_{\mathcal{D}\left(\mathcal{G}_{S}\right)}$ we can define, up to permutations, a class of representative decision sets, $\mathcal{F}_{\mathcal{G}_{S}}(\overline{\mathcal{S}})$ satisfying the following condition:

\footnotetext{
${ }^{5}$ Notice that $\bar{R}=\{\{b \prec c\},\{a\}\}$ and $\bar{R}^{\prime}=\{\{a \prec c\},\{b\}\}$. This means that these social orders are not complete. In the former case only $b$ and $c$ can be compared, while $a$ is not preferred (nor indifferent) to the other two alternatives. In the case of $\bar{R}^{\prime}$ the comparable alternatives are $a$ and $c$, while $b$ remains unrelated to them.

${ }^{6}$ The proofs of the claims can be found in the Appendix.
} 
(i) for each situation $\mathcal{S} \in \overline{\mathcal{S}}$ there exist a permutation $\gamma \in \mathcal{G}_{S}$ such that for each pair $s, t \in S, D(\{s, t\}) \in \mathcal{F}_{\mathcal{G}_{S}}(\overline{\mathcal{S}})$, where $D(\{s, t\})=$ $D^{\mathcal{S}}\left(\left\{\gamma^{-1}(s), \gamma^{-1}(t)\right\}\right)$ and,${ }^{7}$

(ii) there does not exist a permutation $\rho: S \rightarrow S$ such that $D(\{s, t\}), D(\{\rho(s), \rho(t)\}) \in \mathcal{F}_{\mathcal{G}_{S}}(\overline{\mathcal{S}})$ with $D(\{s, t\})=D(\{\rho(s), \rho(t)\})$.

Condition $(i i)$ ensures that each $D(\{s, t\}) \in \mathcal{F}_{\mathcal{G}_{S}}(\overline{\mathcal{S}})$ is uniquely determined, since otherwise we would have for every permutation $\rho: S \rightarrow S$, a class $D(\{\rho(s), \rho(t)\})$ satisfying condition $(i)$. On the other this means that there are different ways of defining $\mathcal{F}_{\mathcal{G}_{S}}(\overline{\mathcal{S}})$, choosing its elements as to satisfy both $(i)$ and $(i i)$. But all the possible alternative characterizations are the same up to permutations of the names of alternatives. We only need to ensure that once chosen one description of the class of representative decisive sets, it remains the same in all ensuing analyses.

Given any situation $\mathcal{S}=\left\langle S, R_{1}, \ldots, R_{n} ; \bar{R}\right\rangle$, its associated preference profile is $\operatorname{prof}_{\mathcal{S}}=\left\langle R_{1}, \ldots, R_{n}\right\rangle$. For the entire class of situations, $\widehat{\mathcal{S}}$, we associate the class $\operatorname{prof}_{\widehat{\mathcal{S}}}=\left\{\operatorname{prof}_{\mathcal{S}}: \mathcal{S} \in \widehat{\mathcal{S}}\right\}$ of all possible preference profiles. $\operatorname{Ord}_{S}$ denotes the class of all possible orderings of $S$. With these definitions at hand we can now study the structure of aggregation functions:

Definition $3 A$ (partial) aggregation function is

$$
\mathbf{f}: \operatorname{prof}_{\widehat{\mathcal{S}}} \rightarrow \operatorname{Ord}_{S}
$$

In words: an aggregation function takes as its argument a profile of preferences over $S$, $\operatorname{prof}_{\mathcal{S}}$, and yields a (partial) ordering of $S .{ }^{8}$ Given the permutation group $\mathcal{G}_{S}, \mathbf{F}_{\mathcal{G}_{S}}$ is the class of aggregation functions such that, given $\gamma \in \mathcal{G}_{S}$, each $\mathbf{f} \in \mathbf{F}_{\mathcal{G}_{S}}$ verifies $\gamma\left(\mathbf{f}\left(\operatorname{prof}_{\mathcal{S}}\right)\right)=\mathbf{f}\left(\operatorname{prof}_{\gamma(\mathcal{S})}\right)$. We will now introduce a social welfare function containing minimal information, that will be shown useful in our analysis:

Definition 4 An aggregation function $\mathbf{f} \in \mathbf{F}_{\mathcal{G}_{S}}$ is said to be prime iff there does not exist an aggregation function $\mathbf{f}^{\prime} \in \mathbf{F}_{\mathcal{G}_{S}}, \mathbf{f}^{\prime} \neq \mathbf{f}$, verifying that $\mathbf{f}^{\prime}\left(\operatorname{prof}_{\mathcal{S}}\right) \neq \emptyset$ for some prof $f_{\mathcal{S}}$ and $\mathbf{f}^{\prime}\left(\operatorname{prof}_{\mathcal{S}}\right) \subseteq \mathbf{f}\left(\operatorname{prof}_{\mathcal{S}}\right)$ for every profile in $\operatorname{prof}_{\widehat{\mathcal{S}}}$.

\footnotetext{
${ }^{7}$ In more technical terms this means that for each $\mathcal{S} \in \overline{\mathcal{S}}$ there exist a permutation $\gamma$, such that $\mathcal{F}_{\mathcal{G}_{S}}(\overline{\mathcal{S}})$ is equivalent modulo $\gamma$ to the class of decision sets of $\mathcal{S}$.

${ }^{8}$ Notice that, on one hand, $\mathbf{f}\left(\operatorname{prof}_{\mathcal{S}}\right)$ may not necessarily coincide with the social relation $\bar{R}$ in the situation $\mathcal{S}$. On the other hand, there might exist at least two elements $s, t \in S$ such that $\mathbf{f}\left(\operatorname{prof}_{\mathcal{S}}\right)(s, t)=\emptyset$.
} 
That is, an aggregation function $\mathbf{f}$ is prime if there is no other function $\mathbf{f}^{\prime}$ able to generate the same results as $\mathbf{f}$ on some profiles of preferences, while it yields no relation for all other profiles (see footnote 8).

To make the description of aggregation functions more precise we need to specify their domains and ranges. So, the domain of an aggregation function $\mathbf{f} \in \mathbf{F}_{\mathcal{G}_{S}}$ is

$\operatorname{Dom}(\mathbf{f})=\left\{\operatorname{prof}_{\mathcal{S}} \in \operatorname{prof}_{\widehat{\mathcal{S}}}: \mathbf{f}\left(\operatorname{prof}_{\mathcal{S}}\right)=\mathbf{f}\left(\operatorname{prof}_{\gamma(\mathcal{S})}\right) \neq \emptyset\right.$, for every $\left.\gamma \in \mathcal{G}_{S}\right\}$

while its range is

$\operatorname{Im}(\mathbf{f})=\left\{R \in \operatorname{Ord}(S):\right.$ there exists $\operatorname{prof}_{\mathcal{S}} \in \operatorname{prof}(\widehat{\mathcal{S}}), \mathbf{f}\left(\operatorname{prof}_{\mathcal{S}}\right)=\mathbf{f}\left(\operatorname{prof}_{\gamma(\mathcal{S})}\right)=R$,

$$
\text { for every } \left.\gamma \in \mathcal{G}_{S}\right\} \cup \emptyset \text {. }
$$

Example 2 Consider two situations:

$$
\mathcal{S}=\left\langle\{a, b, c\},\{a \prec b \prec c\}_{1},\{b \prec c \prec a\}_{2},\{c \prec a \prec b\}_{3} ;\{a \prec b \prec c \prec a\}\right\rangle
$$

and

$$
\mathcal{S}^{\prime}=\left\langle\{a, b, c\},\{a \prec b \prec c\}_{1},\{a \prec b \prec c\}_{2},\{a \prec b \prec c\}_{3} ;\{a \prec b \prec c\}\right\rangle .
$$

These two situations are not equivalent since, for example, $D^{\mathcal{S}}(\{b, c\})=$ $\{1,2\}$ while all the decisive sets of $\mathcal{S}^{\prime}$ include the three agents. So there do not exist a possible permutation $\gamma$ such that $D^{\mathcal{S}}(\{b, c\})=D^{\mathcal{S}^{\prime}}(\{\gamma(b), \gamma(c)\})$.

Consider an aggregation function $\mathbf{f}$ such that

$$
\{a \prec b \prec c \prec a\}=\mathbf{f}\left(\{a \prec b \prec c\}_{1},\{b \prec c \prec a\}_{2},\{c \prec a \prec b\}_{3}\right)
$$

and

$$
\{a \prec b \prec c\}=\mathbf{f}\left(\{a \prec b \prec c\}_{1},\{a \prec b \prec c\}_{2},\{a \prec b \prec c\}_{3}\right) .
$$

A possibility is to consider that $\mathbf{f}$ is the majority rule which, for every pair of alternatives, yields the order among them preferred by the majority of the agents (two over three, in this case). This aggregation function is not prime since for example the unanimous rule that yields only the order that is agreed upon by all the agents can explain also the social outcome in situation $\mathcal{S}^{\prime}$. 
This example shows, in particular, that non-equivalent situations may respond to different aggregation functions (if one at least is not prime). This intuition can be formalized as follows:

Theorem 1 Given $\overline{\mathcal{S}} \in \widehat{\mathcal{S}} / \simeq_{\mathcal{D}\left(\mathcal{G}_{S}\right)}$ there exists a prime aggregation function $\mathbf{f}_{\overline{\mathcal{S}}} \in \mathbf{F}_{\mathcal{G}_{S}}$ such that for any situation $\mathcal{S}=\left\langle S\right.$, prof $\left.f_{\mathcal{S}} ; \bar{R}\right\rangle \in \overline{\mathcal{S}}$ we have that

$$
\mathbf{f}_{\overline{\mathcal{S}}}\left(\operatorname{prof}_{\mathcal{S}}\right)=\bar{R} .
$$

Conversely, for any prime aggregation function $\mathbf{f} \in \mathbf{F}_{\mathcal{G}_{S}}$ there exists a $\overline{\mathcal{S}} \in \widehat{\mathcal{S}} / \simeq_{\mathcal{D}\left(\mathcal{G}_{S}\right)}$ such that

$$
\mathbf{f}\left(\operatorname{prof}_{\mathcal{S}}\right)=\bar{R} \neq \emptyset
$$

for every $\mathcal{S}=\left\langle S, \operatorname{prof}_{\mathcal{S}} ; \bar{R}\right\rangle \in \overline{\mathcal{S}}$.

\section{Aggregation Functions and Partitions}

The relation between equivalence classes in $\widehat{\mathcal{S}} / \simeq_{\mathcal{D}\left(\mathcal{G}_{S}\right)}$ and prime functions can be extended to all aggregation functions in $\mathbf{F}_{\mathcal{G}_{S}}$, according to the following property:

Definition 5 The class of aggregation functions $\mathbf{F}_{\mathcal{G}_{S}}$ is partially ordered by the set-theoretical relation of inclusion among their ranges, $\subseteq$. That is, we say that $\mathbf{f} \preceq \mathbf{f}^{\prime}$ iff for every profile prof $f_{\mathcal{S}}, \mathbf{f}\left(\operatorname{prof}_{\mathcal{S}}\right) \subseteq \mathbf{f}^{\prime}\left(\operatorname{prof}_{\mathcal{S}}\right)$.

This ordering allows us to place any aggregation function at some level in the ensuing hierarchy, and thus detect the prime functions that can jointly generate its outcomes. We will show that this ordering has a parallel in the coarsening ordering of partitions of social situations, showing that each aggregation function as an associated partition. Thus each aggregation function's decision sets can also be obtained as the union of more basic sets of decision sets.

The order $\preceq$ is indeed partial since it is reflexive, antisymmetric and transitive. It is not complete, that is, aggregation functions are not necessarily comparable. On the other hand, since we have not placed any restriction on the cardinality of $S$ nor on the type of allowable social orderings, $\operatorname{Ord}(S)$ can be as big as $2^{|S|}$. What really matters for our argument is that for every possible $\mathbf{f}$, its image is such that $\operatorname{Im}(\mathbf{f}) \subseteq \operatorname{Ord}(S)$. That is, $\mathbf{F}_{\mathcal{G}_{S}}$ is bounded from above. According to Zorn's Lemma we have that every chain 
(linear suborder) in $\left\langle\mathbf{F}_{\mathcal{G}_{S}}, \preceq\right\rangle$ has a maximal element. A similar argument shows that, since $\emptyset$ bounds $\mathbf{F}_{\mathcal{G}_{S}}$ from below, the existence of minimal (prime) aggregation functions is ensured.

Example 3 Let us consider the family of $q$ - quota aggregation functions: for each profile $\left\langle R_{1}, \ldots, R_{n}\right\rangle$ and each $s, t \in S, \mathbf{f}^{q}\left(\left\langle R_{1}, \ldots, R_{n}\right\rangle\right)(s, t)$ iff there exists a subset $I^{q} \subseteq\{1, \ldots, n\}$, such that $t^{9}\left|I^{q}\right|=\left\lceil\frac{q \times n}{100}\right\rceil$ and for each $i \in I^{q}$, $R_{i}(s, t)$. For the decreasing sequence $q \downarrow_{0}^{100}$ we have the following chain:

$$
\mathbf{f}^{100} \preceq \ldots \mathbf{f}^{99} \preceq \ldots \mathbf{f}^{0}
$$

i.e. from the unanimous aggregation function to the "anything goes" function.

The ordering among aggregation functions is reflected in the structure of social situations:

Proposition 2 For any aggregation function $\mathbf{f} \in \mathbf{F}_{\mathcal{G}_{S}}$ there exists a family of equivalence classes $\left\{\overline{\mathcal{S}}^{j}\right\}_{j \in J} \subseteq \widehat{\mathcal{S}} / \simeq_{\mathcal{D}\left(\mathcal{G}_{S}\right)}$ such that

$$
\mathbf{f}\left(\operatorname{prof}_{\mathcal{S}}\right)=\bar{R}
$$

for every $\mathcal{S}=\left\langle S, \operatorname{prof}_{\mathcal{S}} ; \bar{R}\right\rangle \in \cup_{j \in J} \overline{\mathcal{S}}^{j}$.

This indicates that each $\mathbf{f} \in \mathbf{F}_{\mathcal{G}_{S}}$ could be associated to a class in the coarsening of the partition $\widehat{\mathcal{S}} / \simeq_{\mathcal{D}\left(\mathcal{G}_{S}\right)}$. This shows, in turn, that the properties of any aggregation function can be analyzed in terms of the behavior of unions of equivalence classes in the partition $\widehat{\mathcal{S}} / \simeq_{\mathcal{D}\left(\mathcal{G}_{S}\right)}$.

The features of $\left\langle\mathbf{F}_{\mathcal{G}_{S}}, \preceq\right\rangle$ are reflected in the aggregational ordering of $\widehat{\mathcal{S}}$ :

Definition 6 The aggregational ordering of $\widehat{\mathcal{S}}$ is a subclass of partitions of $\widehat{\mathcal{S}},\left\langle\Pi^{\alpha}\right\rangle_{\alpha \geq 0}$, such that $\Pi^{0}=\widehat{\mathcal{S}} / \simeq_{\mathcal{D}\left(\mathcal{G}_{S}\right)}$ and for every pair of indexes $\alpha<\alpha^{\prime}$, $\Pi^{\alpha}$ is a refinement of $\Pi^{\alpha^{\prime}}$, i.e., for every $\pi \in \Pi^{\alpha}$ there exists $\pi^{\prime} \in \Pi^{\alpha^{\prime}}$ such that $\pi \subseteq \pi^{\prime}$.

Again, as in the case of Definition 2, the aggregational ordering is not uniquely defined, but any pair of these possible orderings differ only by a permutation of the names of alternatives. Thus, once chosen one ordering

\footnotetext{
${ }^{9}\lceil r\rceil$ is the least upper integer bound of $r$.
} 
it must be the only one considered in all the subsequent analyses. But which one is chosen is immaterial since the results are independent of the specification of the aggregational ordering. Then we have the following result:

Theorem 2 For every chain $\mathcal{C},\left\langle\mathbf{f}^{0}, \mathbf{f}^{1}, \ldots, \mathbf{f}^{i}\right\rangle$ such that for every pair of indexes $k<k^{\prime}, \mathbf{f}^{k} \preceq \mathbf{f}^{k^{\prime}}$, there exists a subsequence $\left\langle\Pi^{\alpha_{0}}, \Pi^{\alpha_{1}}, \ldots, \Pi^{\alpha_{k}}, \ldots\right\rangle_{k \geq 0}$ of $\left\langle\Pi^{\alpha}\right\rangle_{\alpha \geq 0}$, such that

$$
\Pi^{\alpha_{0}}=\widehat{\mathcal{S}} / \simeq_{\mathcal{D}\left(\mathcal{G}_{S}\right)}=\Pi^{0}
$$

and for every pair $k<k^{\prime}, \alpha_{k}<\alpha_{k^{\prime}}$.

Moreover, if $\mathcal{C}=\left\langle\mathbf{f}^{0}, \mathbf{f}^{1}, \ldots, \mathbf{f}^{\mathcal{C} \mid}\right\rangle$, where $|\mathcal{C}|$ is the length of $\mathcal{C}$, we have that every $\mathbf{f}^{k}$ is supported by a $\pi^{k} \in \Pi^{\alpha_{k}}$.

That is, a chain of functions is associated by a chain of partitions. Furthermore, each function in the chain is supported by a class in the corresponding partition.

As discussed in Section 2, each equivalence class $\overline{\mathcal{S}} \in \widehat{\mathcal{S}} / \simeq_{\mathcal{D}\left(\mathcal{G}_{S}\right)}$ is defined by its decision sets, $\mathcal{F}_{\mathcal{G}_{S}}(\overline{\mathcal{S}})=\{\mathcal{D}(s, t)\}_{s, t \in S}$. Notice that each aggregation function $\mathbf{f}$, associated to a particular $\pi^{\mathbf{f}}$ in a partition $\Pi^{\mathbf{f}}$ of $\widehat{\mathcal{S}}$, is defined in terms of the decision sets corresponding to $\pi^{\mathrm{f}}$.

More precisely, since $\pi^{\mathbf{f}} \subseteq \widehat{\mathcal{S}} / \simeq_{\mathcal{D}\left(\mathcal{G}_{S}\right)}$, there exist a set of indexes $J$ such that $\pi^{\mathbf{f}}=\cup_{j \in J} \overline{\mathcal{S}}^{j}$ and therefore the decision sets of $\pi^{\mathbf{f}}$ are $\cup_{j \in J} \mathcal{F}_{\mathcal{G}_{S}}\left(\overline{\mathcal{S}}^{j}\right)=$ $\cup_{j}\left\{\{\mathcal{D}(s, t)\}_{s, t \in S^{j}}\right\}^{j}$.

An immediate consequence of Theorem 2 is that the latter set, which we call $\mathcal{D E C}(\mathbf{f}),{ }^{10}$ defines completely $\mathbf{f}$. In other words:

Proposition 3 For every chain $\mathcal{C}$ in $\left\langle\mathbf{F}_{\mathcal{G}_{S}}, \preceq\right\rangle, \mathcal{C}=\left\langle\mathbf{f}^{0}, \mathbf{f}^{1}, \ldots, \mathbf{f}^{|\mathcal{C}|}\right\rangle$, there exists a sequence

$$
\mathcal{C}^{\mathcal{D E C}}=\left\langle\mathcal{D E C}\left(\mathbf{f}^{0}\right), \mathcal{D E C}\left(\mathbf{f}^{1}\right), \ldots, \mathcal{D E C}\left(\mathbf{f}^{|\mathcal{C}|}\right)\right\rangle
$$

such that $\mathcal{D E C}\left(\mathbf{f}^{k}\right)$ are the decision sets defining $\mathbf{f}^{k}$.

Moreover, for $k<k^{\prime}, \mathcal{D E C}\left(\mathbf{f}^{k}\right) \subseteq \mathcal{D E C}\left(\mathbf{f}^{k^{\prime}}\right)$. That is, $\mathcal{C}^{\mathcal{D E C}}$ is a chain under set-theoretic inclusion.

${ }^{10}$ That is, $\mathcal{D E C}(\mathbf{f})=\cup_{j}\left\{\{\mathcal{D}(s, t)\}_{s, t \in S^{j}}\right\}^{j}$. 


\section{Social Choice Criteria and Aggregation Func- tions}

Since the structure of aggregation functions is paralleled by the structure of their corresponding decision sets we will concentrate on the latter, as they summarize all the relevant information about the aggregation procedures. To be more precise, we will derive the properties satisfied by any aggregation function up from the characteristics of its corresponding decision sets.

A first step towards that goal is to "normalize" the decision sets corresponding to each element in the partition:

Definition 7 Given an aggregation function $\mathbf{f}$, if $\mathcal{S}$ and $\mathcal{S}^{\prime}$ are in its support, $\operatorname{SUP\mathcal {P}}(\mathbf{f})$, for every $s, t \in S$, there exists a permutation $\gamma \in$ $\mathcal{G}_{S}$ such that either $D^{\mathcal{S}^{\prime}}(\{s, t\}) \subseteq D^{\mathcal{S}}(\{\gamma(s), \gamma(t)\})$ or $D^{\mathcal{S}}(\{s, t\}) \subseteq$ $D^{\mathcal{S}^{\prime}}(\{\gamma(s), \gamma(t)\})$. Then

$$
\begin{gathered}
\mathcal{D}(\{s, t\})=\{\gamma: S \rightarrow S: \\
D^{\mathcal{S}^{\prime}}(\{s, t\}) \subseteq D^{\mathcal{S}}(\{\gamma(s), \gamma(t)\}) \\
\text { or } D^{\mathcal{S}}(\{s, t\}) \subseteq D^{\mathcal{S}^{\prime}}(\{\gamma(s), \gamma(t)\}) ; \\
\text { for every } \left.\mathcal{S}, \mathcal{S}^{\prime} \in \mathcal{S U P \mathcal { P }}(\mathbf{f})\right\}
\end{gathered}
$$

is the set of permutations that either transform the decision set of $\mathbf{f}$ for $s$ and $t$ over $\mathcal{S}$ into a subset or a superset of every decision set for a permutation of $s$ and $t$ over $\mathcal{S U P \mathcal { P }}(\mathbf{f})$.

For each $D(\{s, t\}) \in \mathcal{D E C}(\mathbf{f})$ we obtain a $\mathcal{D}(\{s, t\})$. The quotient of permutations under $\mathcal{D E C}(\mathbf{f})$ is, after choosing a basic $\mathcal{S}^{*} \in \mathcal{S U} \mathcal{P} \mathcal{P}(\mathbf{f}),{ }^{11}$

$\mathbf{D E C}_{\mathcal{G}_{S}}(\mathbf{f})=\left\{\left\langle\mathcal{D}(\{s, t\}), D^{\mathcal{S}^{*}}(\{s, t\})\right\rangle: D^{\mathcal{S}^{*}}(\{s, t\}) \in \mathcal{D E C}(\mathbf{f}), \mathcal{S}^{*} \in \mathcal{S U P} \mathcal{P}(\mathbf{f})\right\}$.

Even if $\mathbf{D E C}(\mathbf{f})$ is more complex than $\mathcal{D E C}(\mathbf{f})$, it allows us to get rid of the complications of using definitions modulo permutations. In fact, this allows us to revert (in a sense) some of the effects of the neutrality applied to obtain the decision sets. With this definition we capture all the possible specific forms of a general aggregation rule.

Furthermore, the appropriate choice of a quotient $\mathcal{S}^{*}$ yields the level in the hierarchy of aggregation functions to which a given $\mathbf{f}$ belongs:

\footnotetext{
${ }^{11}$ We will omit the subscript $\mathcal{G}_{S}$, except when necessary.
} 
Proposition 4 Let $\mathcal{S}^{*} \in \mathcal{S U P P}(\mathbf{f})$ be such that for every $s, t \in S$ and every other situation $\mathcal{S} \in \mathcal{S U P P}(\mathbf{f})$ there exists a permutation $\gamma$ verifying that $D^{\mathcal{S}^{*}}(\{s, t\}) \subseteq D^{\mathcal{S}}(\{\gamma(s), \gamma(t)\})$. Then, if $\mathbf{f}=\mathbf{f}^{j^{*}}, \mathcal{S}^{*} \notin \mathcal{S U P \mathcal { P }}\left(\mathbf{f}^{j}\right)$, for any $j<j^{*}$ in a chain $\left\langle\mathbf{f}^{0}, \mathbf{f}^{1}, \ldots, \mathbf{f}^{j^{*}}\right\rangle$.

This means that all the relevant information about the decision sets of $\mathbf{f}$ is compressed in the decision sets that are not permutations of decision sets for any predecessor $\mathbf{f}^{j}$ in a chain starting from a prime aggregation function.

It is straightforward to see that $\mathbf{D E C}(\mathbf{f}) \subseteq \mathcal{G}_{S} \times 2^{n}$. But this potentially enormous amount of possibilities can be substantially reduced if we note that the second component of $\mathbf{D E C}(\mathbf{f})$ bears most of the information that matters for the analysis of the structure of $\mathbf{f}$. That is, once we have chosen $\mathcal{S}^{*}$ in the support of $\mathbf{f}$, we know from Proposition 4 that the decision sets over other elements in $\mathcal{S U P \mathcal { P }}(\mathbf{f})$ can be obtained by applying a certain set of permutations on the decision sets corresponding to $\mathcal{S}^{*}$.

Then, we can just focus our attention over $\left\{D^{\mathcal{S}^{*}}(\{s, t\}): s, t \in S\right\}$. We define a structure $\mathcal{H}(\mathbf{f})=\langle\{1, \ldots, n\}, \mathbf{D E C}(\mathbf{f})\rangle$, where the individuals are seen as "nodes", while each $D^{\mathcal{S}}(\{s, t\})$ is a "hyperedge" among the nodes, labeled both by the alternatives over which it is defined, $\{s, t\}$ and the class of permutations that preserve its decisiveness, $\mathcal{D}(\{s, t\})$. With this characterization, $\mathcal{H}(\mathbf{f})$ is a labeled hypergraph. ${ }^{12}$

Now that we have found a way to show all the information in a compact form, we will proceed by classifying $\left\{D^{S^{*}}(s, t): s, t \in S\right\}$, and then, by finding some features of this social welfare function $\mathbf{f}$.

Example 4 An instance in which we can easily find some features of the aggregation rule, is when $\mathcal{D E C}(\mathbf{f})$ contains all the sets $Q \subseteq 2^{n}$ such that $|Q| \geq\left\lceil\frac{q \times n}{100}\right\rceil$. In this case, the function involved is the q-quota aggregation rule, because we need at least a q percent of agents to decide over a situation.

$\mathcal{D E C}(\mathbf{f})$ induces a social welfare function, denoted $f_{\mathcal{D E C}(\mathbf{f})}$ as

for all $s, t \in S, R(s, t)$ if and only if exists $D \in \mathcal{D E C}(\mathbf{f})$ such that for all

$$
i \in D, R_{i}(s, t)
$$

Some further definitions will help us to detect properties of $\mathbf{f}$ related to its implicit power structure (i.e. the coalitions of agents that impose the social results):

\footnotetext{
${ }^{12}$ In the case in which each hyperedge has a cardinality of 2 and dropping the labels we obtain the notion of graph as a particular case.
} 
Definition 8 For any set $D$, let $\mathcal{D}$ denote a family of subsets of $D$.

- $\mathcal{D}$ is a prefilter over $D$ if

1. $D \in \mathcal{D}$

2. $A \in \mathcal{D}$ and $A \subseteq B$ implies that $B \in \mathcal{D}$

3. $G$ a finite family of $\mathcal{D}$ implies that $\cap G \neq \emptyset$

- $\mathcal{D}$ is a filter over $D$ if $\mathcal{D}$ is a prefilter and for all $A, B \in \mathcal{D}, A \cap B \in \mathcal{D}$

- $\mathcal{D}$ is an ultrafilter over $D$ if $\mathcal{D}$ is a filter and for all $A \in D, A \in \mathcal{D}$ or $D \backslash A \in \mathcal{D}$

A social welfare function $\mathbf{f}$ is said to be:

- oligarchic if there exists $D \subseteq N$ such that

- every member of $D$ has veto; and

- $D \in \mathcal{D E C}(\mathbf{f})$

- collegial if and only if

$$
K(\mathcal{D E C}(\mathbf{f})) \equiv \bigcap_{D \subset \mathcal{D E C}(\mathbf{f})} D
$$

is nonempty. The set $K(\mathcal{D E C}(\mathbf{f}))$ is called a collegium.

- dictatorial if there exists an agent $i$ (a dictator) such that $R_{i}(s, t)$ implies that $R(s, t)$ for every pair of alternatives $s$ and $t$.

With all these definitions at hand, we have the following result, that just restates Theorem 2.7 in Austen-Smith \& Banks (1998, p. 49) in our setting: ${ }^{13}$

Theorem 3 Let $\mathcal{D E C}(\mathbf{f})$ be the set of decisive coalitions of $\mathbf{f}$. If $\mathcal{D E C}(\mathbf{f})$ is:

1. an ultrafilter, then $f_{\mathcal{D E C}(\mathbf{f})}$ is dictatorial.

\footnotetext{
${ }^{13}$ Notice that the literature always assumes as given the main properties and even the functional form of the social welfare function and then derives its structure of decisive sets to find the implicit power structure. Here instead, we start from nothing else than the social situations and find those structures up from the equivalence among situations.
} 
2. a filter, then $f_{\mathcal{D E C}(\mathbf{f})}$ is oligarchic.

3. a prefilter, then $f_{\mathcal{D E C}(\mathbf{f})}$ is collegial.

In each case, $f_{\mathcal{D E C}(\mathbf{f})}$ is weakly Paretian and independent of irrelevant alternatives.

Let us see some examples that sheds some light on the entire procedure:

Example 5 Consider the following three social situations:

$$
\begin{aligned}
& \mathcal{S}=\left\langle\{a, b, c\},\{a \prec b \prec c\}_{1},\{b \prec c \prec a\}_{2},\{a \prec b \prec c\}_{3} ;\{\{b \prec c\},\{a\}\}\right\rangle, \\
& \mathcal{S}^{\prime}=\left\langle\{a, b, c\},\{b \prec c \prec a\}_{1},\{b \prec a \prec c\}_{2},\{a \prec b \prec c\}_{3} ;\{\{b \prec c\},\{a\}\}\right\rangle
\end{aligned}
$$

and

$$
\mathcal{S}^{\prime \prime}=\left\langle\{a, b, c\},\{b \prec a \prec c\}_{1},\{a \prec c \prec b\}_{2},\{b \prec a \prec c\}_{3} ;\{\{a \prec c\},\{b\}\}\right\rangle
$$

In these situations, the agents order linearly the three alternatives $a, b, c$ and the social outcome is similar: one alternative is preferred to another, while the remaining one does not bear any relation with the other two alternatives. We have that

$$
D^{\mathcal{S}}(\{b, c\})=D^{\mathcal{S}^{\prime}}(\{b, c\})=D^{\mathcal{S}^{\prime \prime}}(\{a, c\})=\{1,2,3\}
$$

It is easy to see that all the other decisive sets are empty. Then, we have that $\mathcal{S} \simeq_{\mathcal{D}} \mathcal{S}^{\prime}$ under $I d_{S}$ and $\mathcal{S} \simeq_{\mathcal{D}} \mathcal{S}^{\prime \prime}$ under (12). The class of its decision sets is:

$$
\mathcal{F}_{\mathcal{G}_{S}}(\overline{\mathcal{S}})=\{1,2,3\}
$$

Maybe if we have more social situations, we can find another class of decisive set. The decision set of $\mathbf{f}$ is:

$$
\mathcal{D E C}(\mathbf{f})=\{1,2,3\}
$$

Finally we find that:

$$
\operatorname{DEC}(\mathbf{f})=\left\{\left\langle I d_{S},\{1,2,3\}\right\rangle,\langle(12),\{1,2,3\}\rangle\right\}
$$

With all these information we can construct the associated labelled hypergraph:

$$
\mathcal{H}(\mathbf{f})=\left\langle\{1,2,3\},\left\{\left\langle I d_{S},\{1,2,3\}\right\rangle,\langle(12),\{1,2,3\}\rangle\right\}\right\rangle
$$

Now we focus on $\mathcal{D E C}(\mathbf{f})$. We can see that it is a filter, so, using the Theorem 3, we have that $f$ is oligarchic. In this specific case, we have the unanimity rule, because the oligarchy is made up of all the society (3 agents). 
Example 6 Consider the following four social situations:

$\mathcal{S}=\left\langle\{a, b, c, d\},\{a \prec b \prec c \prec d\}_{1},\{a \prec b \prec d \prec c\}_{2},\{d \prec a \prec b \prec c\}_{3} ;\{a \prec b \prec c \prec d\}\right\rangle$,

$\mathcal{S}^{\prime}=\left\langle\{a, b, c, d\},\{b \prec a \prec c \prec d\}_{1},\{b \prec a \prec d \prec c\}_{2},\{c \prec a \prec b \prec d\}_{3} ;\{b \prec a \prec c \prec d\}\right\rangle$,

$\mathcal{S}^{\prime \prime}=\left\langle\{a, b, c, d\},\{c \prec a \prec b \prec d\}_{1},\{c \prec a \prec d \prec b\}_{2},\{c \prec b \prec d \prec a\}_{3} ;\{c \prec a \prec b \prec d\}\right\rangle$

and

$\mathcal{S}^{\prime \prime \prime}=\left\langle\{a, b, c, d\},\{a \prec b \prec c \prec d\}_{1},\{a \prec d \prec b \prec c\}_{2},\{c \prec b \prec a \prec d\}_{3} ;\{a \prec b \prec c \prec d\}\right\rangle$

Straightforward calculations indicate that they are not equivalent. We will focus on $\mathcal{D E C}(\mathbf{f})$. If we only consider $\mathcal{S}^{\prime}, \mathcal{S}^{\prime \prime}, \mathcal{S}^{\prime \prime \prime}$, we have that

$$
\mathcal{D E C}(\mathbf{f})=\langle\{1,2\},\{1,3\},\{1,2,3\}\rangle
$$

is a prefilter, so we have that agent 1 belongs to the collegium. But, if we consider $\mathcal{S}$ and $\mathcal{S}^{\prime}$, we have that

$$
\mathcal{D E C}(\mathbf{f})=\langle\{1\},\{1,2\},\{1,3\},\{1,2,3\}\rangle
$$

is an ultrafilter and agent 1 is a dictator. This means that the addition of situation $S$ gives extra power to agent 1. On the other hand $S$ by itself does not allow to obtain any conclusion.

Some intuitions can be drawn from these examples. Depending on $\mathcal{G}_{S}$ some information is lost when considering $\mathcal{H}(\mathbf{f})$, in particular, anything that refers to the name of an alternative. On the other hand, if $\mathcal{D E C}(\mathbf{f})$ has some proper structure, the general criteria satisfied by the social welfare function (like the existence of a collegium, dictator, etc) can still be detected.

In fact, if we consider the large class of voting rules (Austen-Smith \& Banks, 1998 p. 58), which consists of all the aggregation functions that can be generated by their decision sets (assuming that some of the individuals in them have only weak preferences over the alternatives), we have trivially:

Proposition 5 For every voting rule $\mathbf{f}$, we can find $\mathcal{D E C}(\mathbf{f})$ such that $\mathbf{f}=f_{\mathcal{D E C}(\mathbf{f})}$ and thus, $\mathcal{H}(\mathbf{f})$ captures all its relevant properties.

In such cases, then, we seek to write down the properties implied by $\mathcal{H}(\mathbf{f})$. 


\section{Theories up from $\mathcal{H}(\mathbf{f}) \mathrm{s}$}

The criteria satisfied by $\mathcal{H}(\mathbf{f})$ can be conceived as related to the axioms in a formal system that defines the properties of the aggregation function. To make this more precise let us give some definitions:

Definition 9 The formal language for aggregation functions over the set of alternatives $S$ and $n$ agents, $\mathcal{L}_{n}^{S}$, is a two-sorted first order language, and the class of its sentences is $\mathbf{S E N T}\left(\mathcal{L}_{n}^{S}\right) .{ }^{14}$

$A$ theory of an aggregation function on this setting is $\mathcal{T} \subseteq \operatorname{SENT}\left(\mathcal{L}_{n}^{S}\right)$ such that $\mathcal{T}$ is consistent: $\mathcal{T} \forall \perp$. A set of axioms for $\mathcal{T}$ is $\mathcal{A} \subseteq \mathcal{T}$ such that $\mathcal{A} \vdash \mathcal{T}$.

Example 7 Consider the case of the aggregation function $\mathbf{f}$ over three alternatives, which for three agents imposes agent 1's will over the entire group. We can denote this in FOL as:

$$
\phi^{1}: \forall x, y R(1, x, y) \rightarrow \bar{R}(x, y)
$$

where $\bar{R}(x, y)$ is interpreted as the more-than-or-equal preferential ordering imposed by $\mathbf{f}$, on $x, y$, which are variables ranging over the set of alternatives $\{a, b, c\}$, while $R(i, \cdot, \cdot)$ is such that its first argument ranges over the set of agents, while the other two range over the alternatives. If we want to have a theory of $\mathbf{f}$, say $\mathcal{T}^{\mathbf{f}}, \phi^{1}$ must be such that $\phi^{1} \in \mathcal{T}^{\mathbf{f}}$. Moreover, since $\phi^{1}$ characterizes the aggregation function, $\phi^{1} \in \mathcal{A}^{\mathbf{f}}$, i.e. it is an axiom of the theory. On the other hand, it is clear that $\mathbf{f}$ verifies trivially the Pareto condition, which can be represented as:

$$
\phi^{P}: \forall x, y[R(1, x, y) \wedge R(2, x, y) \wedge R(3, x, y)] \rightarrow \bar{R}(x, y)
$$

Then we have that $\phi^{P} \in \mathcal{T}^{\mathrm{f}}$ and, moreover, that $\phi^{1} \vdash \phi^{P}$. Instead, if we have that a formula like:

$$
\phi^{-1}: \forall x, y R(1, x, y) \rightarrow \neg \bar{R}(x, y)
$$

stating that 1 is an antidictator(i.e. what 1 chooses is never chosen by the society), $\phi^{-1} \notin \mathcal{T}^{\mathrm{f}}$, since otherwise $\phi^{1} \wedge \phi^{-1} \vdash \perp$. A weaker version of $\phi^{-1}$, namely

$$
\phi^{-1^{\prime}}: \forall x, y R(1, x, y) \rightarrow \bar{R}(y, x)
$$

is, instead, not inconsistent with $\mathcal{T}^{\mathrm{f}}$.

\footnotetext{
${ }^{14}$ For a general treatment of first-order logic see Smullyan (1995).
} 
The syntactic characterization given in Definition 9 has a model-theoretic counterpart. That is, each sentence in $\mathcal{L}_{n}^{S}$ may be interpreted in such a way that a theory $\mathcal{T} \subseteq \operatorname{SENT}\left(\mathcal{L}_{n}^{S}\right)$ can be seen as a set of claims about the behavior of the constants and relations in the interpretation. If there exists an interpretation $\mathcal{M}$ that satisfies (makes true) every formula of $\mathcal{T}$ it is called a model of the theory. Moreover, if $\mathcal{T}$ is consistent it has at least one model. ${ }^{15}$

In our case, as the previous example shows, the interpretation of symbols in a theory about the behavior of an aggregation function is straightforward: variables and constants range over the sets of individuals and alternatives, $\{1, \ldots, n\}$ and $\mathcal{S}$ respectively, while any atomic expression $R(i, x, y)$ is interpreted as the preference of agent $i$ over $x, y$, i.e. $R_{i}(x, y)$. Finally, if the interpretation is intended to be a model, $\bar{R}$ is to be interpreted as the social preference imposed by $\mathbf{f}$. Therefore, any theory $\mathcal{T}^{\mathbf{f}}$ of a aggregation function $\mathbf{f}$ has each situation $\mathcal{S} \in \mathcal{S U P \mathcal { P }}(\mathbf{f})$ as one of its models. Moreover, if $\mathcal{S}=\left\langle S, R_{1}, \ldots, R_{n} ; \bar{R}\right\rangle \notin \mathcal{S U P \mathcal { P }}(\mathbf{f})$, we have by definition that $\mathbf{f}(\operatorname{prof}(\mathcal{S})) \neq \bar{R}$. Then $\mathcal{S}$ is not a model of $\mathcal{T}^{\mathrm{f}}$.

Given any structure $\mathcal{M}$ we may look for a theory for which $\mathcal{M}$ is a model. If $\mathcal{M}$ has only a finite number of constants and relations defined over them, it is easy to find a finite set of sentences that can be interpreted to yield the true relations in the structure. Things become more interesting if we intend to find a theory corresponding to a given a class of structures $\overline{\mathcal{M}}$. In particular, we will try to find $\mathcal{T}^{\mathrm{f}}$ given $\mathcal{S U P \mathcal { P }}(\mathbf{f})$.

As discussed above, the labeled hypergraph $\mathcal{H}(\mathbf{f})$ represents in summary form the information about $\mathcal{S U P P}(\mathbf{f})$. It indicates the decisive sets for every pair of alternatives and the permutations of agents that transform the decisive sets for a situation into the decisive sets in another situation in $\operatorname{SUPP}(\mathbf{f})$. This information is useful to derive $\mathcal{T}^{\mathbf{f}}$.

As said, we expect to derive $\mathcal{T}^{\mathbf{f}}$ up from $\mathcal{H}(\mathbf{f})$ but first we have to show that this is feasible. Notice that $\mathcal{H}(\mathbf{f})$ yields, applying the permutations indicated in the labels of its hyperedges, a number of families of alternative decision sets over the same set of alternatives. This information can be used to reconstruct a class of social choice situations, namely those in $\mathcal{S U P P}(\mathbf{f})$. Each of those is a model for a collection of first order formulas $\mathcal{C}$. This formulas obtain just translating the corresponding relations described by

\footnotetext{
${ }^{15}$ This is why in Example $7, \phi^{1}$ and $\phi^{-1^{\prime}}$ are not inconsistent: there exist a model where they are both true. In that model the social order is indifferent between any pair of alternatives.
} 
$\mathcal{H}(\mathbf{f})$. Consider $\mathcal{L}_{n}^{S}$ as a two-sorted formal language with formulas built up from the following elements:

- constants of the agent sort: $\bar{I}=\{\overline{1}, \overline{2}, \ldots, \bar{n}\}$,

- constants of the alternative sort: $\bar{A}=\{\bar{s}, \bar{t}, \ldots\}$, such that $|\bar{A}|=|S|$,

- a family of variables of the agent sort, $\hat{I}$, with elements $\hat{i}, \hat{\jmath}, \hat{k}$, etc.,

- a family of variables of the alternative sort, $X$, with elements denoted $x, y$, etc.,

- an individual propositional function, $R(\cdot, \cdot, \cdot)$ that ranges over $\bar{I} \times \bar{A} \times \bar{A}$,

- a social propositional function $\bar{R}(\cdot, \cdot)$ ranging over $\bar{A} \times \bar{A}$,

- a family of functions $\overline{\mathcal{G}}_{S}$ where each $\bar{\gamma} \in \overline{\mathcal{G}}_{S}, \bar{\gamma}: \bar{A} \rightarrow \bar{A}$ is a permutation of constants of the alternative sort,

- a family of functions $\overline{\mathcal{G}}_{n}$ where each $\bar{\rho} \in \overline{\mathcal{G}}_{n}, \bar{\rho}: \bar{I} \rightarrow \bar{I}$ is a permutation of constants of the agent sort,

- the usual connectives and quantifiers of first-order logic.

In this restricted language the information in $\mathcal{H}(\mathbf{f})$ can be immediately expressed as follows. Each hyperedge is, as said, $\mathbf{H}=\left\langle\mathcal{I}, \mathcal{P}_{\mathcal{S}}, \mathbf{G}_{\mathbf{n}}, \mathbf{G}_{\mathbf{S}}\right\rangle$, where $\mathcal{I}$ is the set of agents connected by the hyperedge, $\mathcal{P}_{\mathcal{S}} \in \mathcal{S} \times \mathcal{S}$ is the pair of states over which those agents are decisive in $\mathcal{D E C}(\mathbf{f})$. Finally $\mathbf{G}_{\mathbf{n}} \subseteq \mathcal{G}_{\mathbf{n}}$ and $\mathbf{G}_{\mathbf{S}} \subseteq \mathcal{G}_{\mathbf{S}}$ are the families of permutations that preserve the decisiveness of $\mathcal{I}$ over $\mathcal{P}_{\mathcal{S}}$. Then we can obtain a family of first order sentences in $\operatorname{SENT}\left(\mathcal{L}_{n}^{S}\right)$, which will be denoted $\Phi^{\mathrm{f}}$ :

Definition 10 For each hyperedge $\mathbf{H}$ of $\mathcal{H}(\mathbf{f})$, if $\mathcal{P}_{S}=\langle a, b\rangle$ then, for every $i \in \mathcal{I}$, every $\rho \in \mathbf{G}_{\mathbf{n}}$ and every $\gamma \in \mathbf{G}_{\mathbf{S}}$, the sentence

$$
\phi: R(\bar{\rho}(i), \bar{\gamma}(a), \bar{\gamma}(b)) \rightarrow \bar{R}(\bar{\gamma}(a), \bar{\gamma}(b))
$$

is included in $\Phi^{\mathrm{f}}$.

Each $\mathcal{S} \in \mathcal{S U P \mathcal { P }}(\mathbf{f})$ constitutes an interpretation of $\Phi^{\mathrm{f}}$ by construction. This family is the basis on which we will define $\mathcal{T}^{\mathbf{f}}$ as:

$$
\mathcal{T}^{\mathbf{f}}=\left\{\mu \in \operatorname{SENT}\left(\mathcal{L}_{n}^{S}\right): \Phi^{\mathbf{f}} \vdash \mu\right\}
$$


Notice that the number of formulas in $\mathcal{T}^{\mathrm{f}}$ is infinite. Even if $S$ is finite, if any $\mathcal{S} \in \mathcal{S U P P}(\mathbf{f})$ is an interpretation for a formula $\phi \in \mathcal{T}^{\mathrm{f}}$, it also interprets $\phi \vee \psi$, for any first order sentence $\psi$. On the other hand, since we assume that each $\mathcal{S} \in \mathcal{S U P P}(\mathbf{f})$ is a model for $\mathcal{T}^{\mathrm{f}}$ it follows that it is consistent.

On the other hand, consider two different situations $\mathcal{S}$ and $\mathcal{S}^{\prime}$ drawn from $\mathcal{S U P \mathcal { P }}(\mathbf{f})$. Since their corresponding decision sets are the same up to permutations as defined in $\mathbf{H}$ of $\mathcal{H}(\mathbf{f})$, there exists an isomorphism between $\mathcal{S}$ and $\mathcal{S}^{\prime}$, through the corresponding permutations. Technically, $\mathcal{S}$ and $\mathcal{S}^{\prime}$ are said elementarily equivalent models of $\mathcal{T}^{\mathrm{f}}$. A well known result in model theory is (Stigum 1990):

Completeness Theorem: Let $\mathcal{T}$ be a consistent first order theory with language $\mathcal{L}$. Then, $\mathcal{T}$ is complete if and only if any two models of $\mathcal{T}$ are elementarily equivalent.

Even if $\mathcal{T}^{\mathrm{f}}$ is well defined and has the desirable metamathematical properties of consistency and completeness, we are interested in finding an axiomatization for $\mathbf{f}$, which means a minimal number of formulas $\mathcal{A}^{\mathrm{f}} \subseteq \mathcal{T}^{\mathrm{f}}$ such that for every $\mu \in \mathcal{T}^{\mathbf{f}}, \mathcal{A}^{\mathbf{f}} \vdash \mu$. We say that $\mathcal{A}^{\mathbf{f}}$ is the set axioms of $\mathcal{T}^{\mathrm{f}} .16$

Two different cases may arise. One is when $S$ and $n$ are finite and the other when either one is infinite. Each involves a different treatment.

\subsection{Finite Number of Agents and Alternatives}

In this case $\Phi^{\mathbf{f}}$ is finite. It is easy to check out, by the properties of the hyperedge $\mathbf{H}$ from which it was drawn, whether a given sentence $\phi$, $R(i, a, b) \rightarrow \bar{R}(a, b)$ is verified by every $\rho \in \mathcal{G}_{n}$ and every $\gamma \in \mathcal{G}_{S}$ or not. Then

- If $\phi$ is verified by every $\rho \in \mathcal{G}_{n}$ and every $\gamma \in \mathcal{G}_{S}$, then define $\phi^{\forall \forall}$ : $\forall \bar{\rho} \forall \bar{\gamma}[R(\bar{\rho}(i), \bar{\gamma}(a), \bar{\gamma}(b)) \rightarrow \bar{R}(\bar{\gamma}(a), \bar{\gamma}(b))] .{ }^{17}$

- If $\phi$ is verified by every $\rho \in \mathcal{G}_{n}$ and by many but not all $\gamma \in \mathcal{G}_{S}$, define $\phi^{\forall \exists}: \forall \bar{\rho} \exists \bar{\gamma}[R(\bar{\rho}(i), \bar{\gamma}(a), \bar{\gamma}(b)) \rightarrow \bar{R}(\bar{\gamma}(a), \bar{\gamma}(b))]$.

\footnotetext{
${ }^{16}$ We will restrict the meaning of $\mathcal{A}^{\mathbf{f}}$ to be the non-logical axioms of $\mathcal{T}^{\mathrm{f}}$. The logical axioms are those of first-order logic.

${ }^{17}$ Notice that this sentence is still first order, since the quantifiers do not range over propositional (i.e. truth-valued) functions.
} 
- If $\phi$ is verified by every $\gamma \in \mathcal{G}_{S}$ and by not all $\rho \in \mathcal{G}_{n}$, define $\phi^{\exists \forall}$ : $\forall \bar{\gamma} \exists \bar{\rho}[R(\bar{\rho}(i), \bar{\gamma}(a), \bar{\gamma}(b)) \rightarrow \bar{R}(\bar{\gamma}(a), \bar{\gamma}(b))]$.

- If $\phi$ is verified by some but not all $\rho \in \mathcal{G}_{n}$ and $\gamma \in \mathcal{G}_{S}$, define $\phi^{\exists}$ : $\exists \bar{\rho} \exists \bar{\gamma}[R(\bar{\rho}(i), \bar{\gamma}(a), \bar{\gamma}(b)) \rightarrow \bar{R}(\bar{\gamma}(a), \bar{\gamma}(b))]$.

This means that each hyperedge $\mathbf{H}$ can be represented by a sentence, denoted by $\phi_{\mathbf{H}}$ (either $\phi_{\mathbf{H}}^{\forall}, \phi_{\mathbf{H}}^{\forall \exists}, \phi_{\mathbf{H}}^{\exists}$ or $\phi_{\mathbf{H}}^{\exists}$ ). Of course, some information is lost, but the decision structure is kept.

By a similar examination of the class $\left\{\phi_{\mathbf{H}}\right\}_{\mathbf{H} \in \mathcal{H}(\mathbf{f})}$, we obtain eight possible cases of the form

$$
\phi^{\triangle}: \triangle_{\hat{I}^{\hat{1}}} \triangle_{X}^{1} x \triangle_{X}^{2} y \phi_{\mathbf{H}}
$$

where $\triangle_{\hat{I}}, \triangle_{X}^{1}$ and $\triangle_{X}^{2}$ are either $\forall$ or $\exists$.

It is immediate that:

Proposition 6 The quantified sentences $\phi^{\triangle}$ and $\phi_{\mathbf{H}}$ entail the entire set of sentences $\Phi^{\mathrm{f}}$ :

$$
\left\{\phi^{\triangle}\right\} \cup\left\{\phi_{\mathbf{H}}\right\}_{\mathbf{H} \in \mathcal{H}(\mathbf{f})} \vdash \Phi^{\mathbf{f}} .
$$

This result indicates that $\mathcal{A}^{\mathbf{f}}=\left\{\phi^{\triangle}\right\} \cup\left\{\phi_{\mathbf{H}}\right\}_{\mathbf{H} \in \mathcal{H}(\mathbf{f})}$ provides a finite axiomatization of $\mathcal{T}^{\mathrm{f}}$. This family of axioms $\mathcal{A}$ conveys the minimal essential information about social situations, while the full information is provided by $\mathcal{T}^{\mathrm{f}}$.

The set of axioms $\mathcal{A}^{\mathbf{f}}$ can be seen in the light of the following classification (Fishburn 1987): ${ }^{18}$

- Structural conditions. They prescribe conditions on the domain of aggregation (individuals, alternatives and profiles). The structural conditions just indicate the elements on which the aggregation procedure will be applied. Easy examples are the requirement that all individual preferential orderings have to be weak orders or that the number of individuals has to be larger than 2 .

- Existential conditions. They prescribe the existence of certain results under an aggregation procedure. So, for example, an existential condition is that there should not exist an individual such that for every

\footnotetext{
${ }^{18}$ They capture the features of voting rules, which are completely described by $\mathcal{H}(\mathbf{f})$, as indicated in Proposition 5.
} 
profile her preferences coincide with those that result from the aggregation procedure. ${ }^{19}$

- Universal conditions. They specify general aspects of the aggregation procedure. The universal conditions can be further classified as intraprofile conditions, which involve no comparisons among profiles or interprofile ones. An example of intraprofile conditions are the Pareto condition, which indicates that if every individual in a profile prefers more one alternative over another one, this feature must result also in the social ordering. An interprofile condition is, instead, the independence of irrelevant alternatives, which prescribes that if in two profiles the preferences of the agents with respect to any pair of alternatives are the same, then the order over these alternatives in the respective social orderings must coincide.

It follows that:

- The joint characterization of $S, n$ and the corresponding $\mathcal{G}_{S}$ and $\mathcal{G}_{n}$ yields the structural conditions on the aggregation function $\mathbf{f}$.

- The sentences $\phi_{\mathbf{H}}$ yield the interprofile conditions.

- The sentences $\phi^{\triangle}$ summarize both the interprofile and intraprofile conditions.

This indicates that to disentangle the intraprofile from the interprofile conditions, more information is needed, which can be found in $\Phi^{\mathbf{f}}$. The family of axioms $\mathcal{A}^{\mathbf{f}}$, provides only the most general properties of $\mathbf{f}$ while others can be derived from them. A simple example shows this:

Example 8 (Examples 5 and 6 revisited) Consider either one of the unlabeled hypergraphs, say $\mathcal{H}(\mathbf{f})=\langle 3,\{\{1\},\{1,2\},\{1,3\}\}\rangle$. It constitutes, as said, an ultrafilter, i.e. every subset $A \subseteq\{1,2,3\}$ either $A \in \mathcal{H}(\mathbf{f})$ or $A^{C} \in \mathcal{H}(\mathbf{f})$, where $A^{C}=\{1,2,3\} \backslash A$. Since $\{1,2,3\}$ is finite, $\mathcal{H}(\mathbf{f})$ is a principal ultrafilter. That is, $\cap \mathcal{H}(\mathbf{f})$ is a singleton. The element in this singleton denotes a dictator. ${ }^{20}$ Then, the only $\phi^{\triangle}$ axiom that can be derived is $\phi: \exists \hat{\imath} \forall \mathbf{x}, \mathbf{y} \mathbf{R}(\hat{\imath}, \mathbf{x}, \mathbf{y}) \rightarrow \overline{\mathbf{R}}(\mathbf{x}, \mathbf{y})$. In fact, $\mathcal{A}^{\mathbf{f}}=\{\phi\}$. Notice that the

\footnotetext{
${ }^{19}$ Notice that this non-dictatorship condition can be rewritten as to become the claim of the existence of a pair of alternatives which for every individual and every profile verify that the social outcome differs from the individual orderings.

${ }^{20}$ See Kirman \& Sondermann (1972), Brown (1974) or Hansson (1976).
} 
variable î might be interpreted as being either one of the possible agents, 1,2 or 3 . That is, in this first order representation the information about the actual dictator is lost. Despite this, and given the knowledge already obtained, it follows that the social choice implicit in the example can be implemented in a dominant strategies equilibrium.

On the other hand, to get a theory that just implies the $\Phi^{\mathrm{f}}$, instead of one as general as $\mathcal{A}^{\mathrm{f}}$, we can just take $\Phi^{\mathrm{f}}$ as the class of our axioms. Since they are finite, the axiomatization is of course also finite. But this procedure has a huge cost associated to it: all the advantages of extracting intensional from extensional information are lost. It is as if we were running a statistical procedure which obtains an exact characterization of the sample by just enumerating its elements!

\subsection{Infinite Number of Agents or Alternatives}

As usual in social-theoretic (and more generally in economic-theoretic) analyses, there is an interest in assessing outcomes when the number of individuals is large. The formal treatment of those cases is based on identifying a "large" number of agents with a (countably) infinite number of them, usually yielding different results that in the finite case (Kirman and Sondermann, 1972). Notice that the language needed is no longer finitary, because of the number of constants required to represent the infinite elements in the domain. While this is a handicap, some results will allow us to recover a characterization of the set of axioms $\mathcal{A}^{\mathrm{f}}$ similar to the presentation in the previous section. Notice that those axioms are expressed with just a few variables. Therefore, we can keep using a finitary fragment of $\mathcal{L}_{n}^{S}$ with the proviso that it will be used only to write down $\mathcal{A}^{\mathrm{f}}$.

Recall that $\mathcal{H}(\mathbf{f})$ is a hypergraph. We seek a family of sentences $\mathcal{A}^{\mathbf{f}}$ that can be satisfied by $\mathcal{H}(\mathbf{f})$ :

Definition $11 \mathcal{A}^{\mathbf{f}}$ is satisfied if and only if there exists $\sigma=$ $\left\langle\mathcal{I}, \mathcal{P}_{\mathcal{S}}, \mathbf{G}_{\mathbf{n}}, \mathbf{G}_{\mathbf{S}}\right\rangle$, where $\mathcal{I} \subseteq I$ is a set of agents, $\mathcal{P}_{\mathcal{S}} \in \mathcal{S} \times \mathcal{S}$ is a pair of states, while $\mathbf{G}_{\mathbf{n}} \subseteq \mathcal{G}_{\mathbf{n}}$ and $\mathbf{G}_{\mathbf{S}} \subseteq \mathcal{G}_{\mathbf{S}}$ are permutations over $n$ and $S$, that verifies (Kolany 1993):

- consistency: $\mathbf{H} \subset \sigma$ for $n o \mathbf{H} \in \mathcal{H}(\mathbf{f})$,

- transversality: $\sigma \cap \mathcal{I N} \mathcal{T}(\alpha) \neq \emptyset$, for every sentence $\alpha \in \mathcal{A}^{\mathbf{f}}$, where $\mathcal{I N} \mathcal{T}(\alpha)$ is a hyperedge that yields a true interpretation of $\alpha$. 
The notion of satisfaction over hypergraphs is in fact equivalent to the existence of a system of distinct representatives, i.e. to the existence of a transversal $\sigma$ that has an element from each hyperedge. The existence of such a $\sigma$ is ensured by the following result:

Theorem(Hurwicz \& Reiter 2001): $\mathcal{H}(\mathbf{f})$ has a transversal $\sigma$ if and only if $\mathcal{H}(\mathbf{f})$ is generated by a self-belonging correspondence $\Upsilon: \cup \mathcal{H}(\mathbf{f}) \rightarrow \bigcup \mathcal{H}(\mathbf{f})$.

Two are the conditions on $\Upsilon: \cup \mathcal{H}(\mathbf{f}) \rightarrow \cup \mathcal{H}(\mathbf{f})$ that may ensure the existence of $\sigma$ :

- generation: for every $\mathbf{H} \in \mathcal{H}(\mathbf{f})$, there exists $\theta \in \bigcup \mathcal{H}(\mathbf{f})$ verifying that $\mathbf{H}=\boldsymbol{\Upsilon}(\theta)$

- self-belonging: for every $\theta \in \bigcup \mathcal{H}(\mathbf{f}), \theta \in \Upsilon(\theta)$.

In fact, it follows that:

Proposition 7 There exists $\Upsilon$ that ensures the existence of a transversal $\sigma$.

Finally:

Lemma 1 There exists a set of sentences $\mathcal{A}^{\mathbf{f}}$ in $\mathcal{L}_{n}^{S}$ that is satisfied by the transversal $\sigma$. Moreover, each $\alpha \in \mathcal{A}^{\mathbf{f}}$ can be either $\phi_{\mathbf{H}}^{\forall}, \phi_{\mathbf{H}}^{\forall}, \phi_{\mathbf{H}}^{\exists}, \phi_{\mathbf{H}}^{\exists}$ or $\phi^{\triangle}$.

Therefore, as in the finite case, a set of axioms can be obtained for a potential theory $\mathcal{T}^{\mathfrak{f}}$. However, as in the former instance, the axioms of $\mathcal{A}^{\mathbf{f}}$, while conveying the general properties of $f$, do not pinpoint their precise details. Recalling that $\Phi^{\mathbf{f}}$ is now an infinite enumeration of sentences without quantifiers, we could apply the following theorem (Shoenfield 1967): Theorem(Eos-Tarski) A theory $\mathcal{T}$ is equivalent to a class of quantifier-free sentences $\Phi$ if and only if every substructure of a model of $\mathcal{T}$ is a model of $\mathcal{T}$.

This means, in our case that a $\mathcal{T}^{\mathrm{f}}$ with precisely those sentences in $\Phi^{\mathrm{f}}$ and only those, must be such that its models have a "Russian doll" structure. That is, for each model its substructures are also models. As in the finite case, if we take the axioms of $\mathcal{T}^{\mathrm{f}}$ to be $\Phi^{\mathrm{f}}$, we have a trivial instance of Łos-Tarski's theorem. But in this case, added to the trivial identification of 
the intensional characterization with the extensional enumeration, we have that the axiom system will no longer be finite. ${ }^{21}$

\section{Conclusions}

In this paper we presented an exercise in inductive reasoning, namely, on how to infer the criteria verified by social welfare functions, up from the observable behavior of their decision sets. We have shown that social welfare functions keep a close relation with partitions of social choice situations. Moreover, each social welfare function yields a corresponding labeled hypergraph.

As usual in inductive inference, the information extracted by the procedure fleshes out the specific details of the characterization of the functions, leaving only their bare bones. By doing this, it erases the differences among similar functions, but at the same time it yields what we entertained originally to find: a few properties that are satisfied by them.

We understand that it can be very restrictive to ask the decisive sets to be prefilters, filters or ultrafilters. We leave out of the analysis many other potential structures by doing this. But if we continue studying them as labeled hypergraphs, we can loosen the hypotheses. In particular, these hypergraphs can be used to represent nerves of coverings by sets of agents, associated to some decisions. This, in turn, may allow the connection between the combinatorial and the topological analysis of social choices (Chichilnisky 1980, Baryshnikov 1997, Lauwers 2000).

On the other hand, even with these shortcomings, this approach provides a tool for the elicitation of the information hidden in salient cases. Once generalized, it may help to assess the possibility of implementing the implicit social choice rule described in those cases.

This research points out, also, to a more general line of inquiry, namely the extraction of intensional information up from an extensional description in partitional form. While the method described is not independent from the underlying problem, many of its features are modular and could be applied to other problems in Economic Theory.

\section{References}

Arrow, K.: Social Choice and Individual Values, Wiley, New York, 1951.

\footnotetext{
${ }^{21}$ In fact, nothing in the theorem ensures that the axiomatization will be finite.
} 
Austen-Smith, D. and Banks, J.: Positive Political Theory I: Collective Preference, The University of Michigan Press, Ann Arbor MI, 1998.

Baryshnikov, Y.: Topological and Discrete Social Choice: In a Search of a Theory, Social Choice and Welfare 1997, 14: 199-209.

Brown, D.: An Approximate Solution to Arrow's Problem, Journal of Economic Theory 1974, 9: 375-383.

Campbell, D. and Kelly, J.: Preference Aggregation, Mathematica Japonica 1997, 45: 573-593.

Chichilnisky, G.: Social Choice and the Topology of Spaces of Preferences, Advances in Mathematics 1980, 37: 165-176.

Fishburn, P.:Interprofile Conditions and Impossibility, Harwood Academic Publishers, Chur (Switzerland), 1987.

Hansson, B.: The Existence of Group Preferences, Public Choice 1976, 28: 89-98.

Hurwicz, L.: Optimality and Informational Efficiency in Resource Allocation Processes, in K. Arrow, S. Karlin and P. Suppes (eds.), Mathematical Methods in the Social Sciences, Stanford University Press, Stanford CA, 1960.

Hurwicz, L. and Reiter, S.: Transversals, Systems of Distinct Representatives, Mechanism Design and Matching, Review of Economic Design 2001, 6: $289-304$.

Kirman, A. and Sondermann, D.: Arrow's Theorem, Many Agents and Invisible Dictators, Journal of Economic Theory 1972, 5: 267-277.

Kolany, A.: On the Logic of Hypergraphs, in Computational Logic and Proof Theory, Springer-Verlag, Berlin, 1993.

Lauwers, L. and Van Liedekerke, L.: Ultraproducts and Aggregation, Journal of Mathematical Economics 1995, 24: 217-237. 
Lauwers, L.: Topological Social Choice, Mathematical Social Sciences 2000, 40: $1-39$.

Mount, K. and Reiter, S.: The Informational Size of Message Spaces, Journal of Economic Theory 1974, 8: 161-192.

Reiter, S.: Information and Performance in the (New) $)^{2}$ Welfare Economics, American Economic Review 1977, 67: 226-234.

Sen, A.: Collective Choice and Social Welfare, Holden Day, San Francisco, CA, 1970.

Shoenfield, J.: Mathematical Logic, Addison-Wesley, Reading, MA, 1967.

Smullyan, R.: First-Order Logic, Dover, New York, 1995.

Stigum, B.: Toward a Formal Science of Economics, MIT Press, Cambridge, MA, 1990.

\section{Appendix}

Proof of Proposition 1 It is immediate from the definition of $\simeq_{\mathcal{D}\left(\mathcal{G}_{S}\right)}$ that for any situation $\mathcal{S}, \mathcal{S} \simeq_{\mathcal{D}\left(\mathcal{G}_{S}\right)} \mathcal{S}$, just taking the identity permutation $I d_{S}$, which belongs to $\mathcal{G}_{S}$ by (A2). That is, $\simeq_{\mathcal{D}\left(\mathcal{G}_{S}\right)}$ is reflexive. To see that it is symmetric, assume $\mathcal{S} \simeq_{\mathcal{D}\left(\mathcal{G}_{S}\right)} \mathcal{S}^{\prime}$ under a $\gamma \in \mathcal{G}_{S}$. Then, by (A3), $\gamma^{-1} \in \mathcal{G}_{S}$, and thus $\mathcal{S}^{\prime} \simeq_{\mathcal{D}\left(\mathcal{G}_{S}\right)} \mathcal{S}$. On the other hand, when $\mathcal{S} \simeq_{\mathcal{D}\left(\mathcal{G}_{S}\right)} \mathcal{S}^{\prime}$ and $\mathcal{S}^{\prime} \simeq_{\mathcal{D}\left(\mathcal{G}_{S}\right)} \mathcal{S}^{\prime \prime}$ we have that there exist permutations $\gamma, \gamma^{\prime} \in \mathcal{G}_{S}$ such that for each $s, t \in S$

$$
\begin{gathered}
D^{\mathcal{S}}(\{s, t\})=D^{\mathcal{S}^{\prime}}(\{\gamma(s), \gamma(t)\}) \text { and } \\
D^{\mathcal{S}^{\prime}}(\{\gamma(s), \gamma(t)\})=D^{\mathcal{S}^{\prime \prime}}\left(\left\{\gamma^{\prime} \circ \gamma(s), \gamma^{\prime} \circ \gamma(t)\right\}\right)
\end{gathered}
$$

That is

$$
D^{\mathcal{S}}(\{s, t\})=D^{\mathcal{S}^{\prime \prime}}\left(\left\{\gamma^{\prime} \circ \gamma(s), \gamma^{\prime} \circ \gamma(t)\right\}\right)
$$

Since, by $(A 1) \gamma^{\prime} \circ \gamma \in \mathcal{G}_{S}, \mathcal{S} \simeq_{\mathcal{D}\left(\mathcal{G}_{S}\right)} \mathcal{S}^{\prime \prime}$. That is, $\simeq_{\mathcal{D}\left(\mathcal{G}_{S}\right)}$ is transitive. 
Proof of Theorem $\mathbf{1} \Rightarrow$ ) Given $\overline{\mathcal{S}} \in \widehat{\mathcal{S}} / \simeq_{\mathcal{D}\left(\mathcal{G}_{S}\right)}$ consider the family of its decision sets, $\mathcal{F}_{\mathcal{G}_{S}}(\overline{\mathcal{S}})$ and define a function $\mathbf{f}_{\overline{\mathcal{S}}}$ as follows. For each profile $\left\langle R_{1}, \ldots, R_{n}\right\rangle$ and for each pair $s, t \in S$, consider the decisive set $D(\{s, t\}) \in \mathcal{F}_{\mathcal{G}_{S}}(\overline{\mathcal{S}})$. Then, for every $\gamma \in \mathcal{G}_{S}$ :

$$
\mathbf{f}\left(\operatorname{prof}_{\gamma(\mathcal{S})}\right)=R(\gamma(s), \gamma(t)) \text { iff for every } i \in D(\{s, t\}), R_{i}(\gamma(s), \gamma(t)) .
$$

By the definition of $\mathcal{F}_{\mathcal{G}_{S}}(\overline{\mathcal{S}}), \mathbf{f}_{\overline{\mathcal{S}}} \in \mathbf{F}_{\mathcal{G}_{S}}$. Moreover, if for every $\mathcal{S}=$ $\left\langle S, R_{1}, \ldots, R_{n} ; \bar{R}\right\rangle \notin \overline{\mathcal{S}}$ we define $\mathbf{f}_{\overline{\mathcal{S}}}\left(R_{1}, \ldots, R_{n}\right)=\emptyset$, it is immediate that $\mathbf{f}_{\overline{\mathcal{S}}}$ is prime.

$\Leftarrow)$ Consider the class of situations with nonempty social orders which can be seen as defined by an arbitrary prime aggregation function $\mathbf{f} \in \mathbf{F}_{\mathcal{G}_{S}}$, $\widehat{\mathcal{S}}^{\mathbf{f}}=\left\{\mathcal{S} \in \widehat{\mathcal{S}}: \mathcal{S}=\left\langle S, R_{1}, \ldots, R_{n} ; \bar{R}\right\rangle, \mathbf{f}\left(R_{1}, \ldots, R_{n}\right)=\bar{R} \neq \emptyset\right\} . \quad A s-$ sume, by way of contradiction, that there exist two equivalence classes $\overline{\mathcal{S}}, \overline{\mathcal{S}}^{\prime} \in \widehat{\mathcal{S}} / \simeq_{\mathcal{D}\left(\mathcal{G}_{S}\right)}$ such that $\widehat{\mathcal{S}}^{\mathbf{f}} \cap \overline{\mathcal{S}} \neq \emptyset$ and $\widehat{\mathcal{S}}^{\mathbf{f}} \cap \overline{\mathcal{S}}^{\prime} \neq \emptyset$. Consider two situations $\mathcal{S} \in \widehat{\mathcal{S}}^{\mathbf{f}} \cap \overline{\mathcal{S}}$ and $\mathcal{S}^{\prime} \in \widehat{\mathcal{S}}^{\mathbf{f}} \cap \overline{\mathcal{S}}^{\prime}$. Then, $\mathcal{S}$ and $\mathcal{S}^{\prime}$ are not equivalent. This can happens for two reasons: for every permutations $\gamma$ there exists at least a pair $s, t \in S$ such that either

$$
\text { - } D^{\overline{\mathcal{S}}}(\{s, t\}) \neq\left(D^{\overline{\mathcal{S}}^{\prime}}\left(\left\{\gamma^{-1}(s), \gamma^{-1}(t)\right\}\right)\right)
$$

or

- either $\bar{R}(s, t)$ or $\bar{R}^{\prime}(\gamma(s), \gamma(t))$ is not defined.

Let us analyze the latter case. Without loss of generality, assume that $\mathcal{S}=\left\langle S, R_{1}, \ldots, R_{n} ; \bar{R}\right\rangle$ and $\mathcal{S}^{\prime}=\left\langle S, R_{1}^{\prime}, \ldots, R_{n}^{\prime} ; \bar{R}^{\prime}\right\rangle$ are such that $R_{i} \equiv R_{i}^{\prime}$ for $i=1, \ldots, n$ while $\bar{R}(s, t)$ and not $\bar{R}^{\prime}(\gamma(s), \gamma(t)) \quad\left(\right.$ nor $\left.\bar{R}^{\prime}(\gamma(t), \gamma(s))\right)$, in particular for $\gamma \equiv I d_{S}$. Since $\mathcal{S}, \mathcal{S}^{\prime} \in \widehat{\mathcal{S}}^{\mathbf{f}}, \bar{R}=\mathbf{f}\left(R_{1}, \ldots, R_{n}\right)$ and $\bar{R}^{\prime}=\mathbf{f}\left(R_{1}, \ldots, R_{n}\right)$. But then, since we assumed that $\bar{R} \not \equiv \bar{R}^{\prime}$ we have $a$ contradiction because $\mathbf{f}$ is assumed to be a function.

Now suppose that $D^{\overline{\mathcal{S}}}(\{s, t\}) \neq D^{\overline{\mathcal{S}}^{\prime}}\left(\left\{\gamma^{-1}(s), \gamma^{-1}(t)\right\}\right)$ for at least a pair of alternatives, $s$ and $t$. According to the $\Rightarrow$ part of this proof there exist two prime aggregation functions $\mathbf{f}^{\prime}$ and $\mathbf{f}^{\prime \prime}$ in $\mathbf{F}_{\mathcal{G}_{S}}$ such that $\mathbf{f}^{\prime}\left(R_{1}, \ldots, R_{n}\right)=\bar{R}$ and $\mathbf{f}^{\prime \prime}\left(R_{1}, \ldots, R_{n}\right)=\bar{R}^{\prime}$. On the other hand we have that $\mathbf{f}^{\prime}\left(R_{1}, \ldots, R_{n}\right) \subseteq$ $\mathbf{f}\left(R_{1}, \ldots, R_{n}\right)$ and $\mathbf{f}^{\prime \prime}\left(R_{1}, \ldots, R_{n}\right) \subseteq \mathbf{f}\left(R_{1}, \ldots, R_{n}\right)$, but this is absurd since f is prime. This shows that there exists an equivalence class $\overline{\mathcal{S}} \in \widehat{\mathcal{S}} / \simeq_{\mathcal{D}\left(\mathcal{G}_{S}\right)}$, such that $\widehat{\mathcal{S}}^{\mathbf{f}} \subseteq \overline{\mathcal{S}}$.

Now assume that there exists a situation $\mathcal{S} \in \overline{\mathcal{S}}$ and $\mathcal{S} \notin \widehat{\mathcal{S}}^{\mathrm{f}}$. This means that if $\mathcal{S}$ is $\left\langle S, R_{1}, \ldots, R_{n} ; \bar{R}\right\rangle$, with $\bar{R} \not \equiv \mathbf{f}\left(R_{1}, \ldots, R_{n}\right)$, there must exist a situation $\mathcal{S}^{\prime}=\left\langle S, R_{1}, \ldots, R_{n} ; \mathbf{f}\left(R_{1}, \ldots, R_{n}\right)\right\rangle \in \widehat{\mathcal{S}}^{\mathbf{f}}$. But then, for 
every permutation $\gamma$, there must exist at least a pair $s, t \in S$ such that $D^{\mathcal{S}}(\{s, t\}) \neq D^{\mathcal{S}^{\prime}}\left(\left\{\gamma^{-1}(s), \gamma^{-1}(t)\right\}\right)$. Absurd, since $\mathcal{S}$ and $\mathcal{S}^{\prime}$ are both in $\overline{\mathcal{S}}$. Therefore $\widehat{\mathcal{S}}^{\mathbf{f}}=\overline{\mathcal{S}}$.

Proof of Proposition $\mathbf{2}$ For every aggregation function $\mathbf{f}$ there exists a family $\left\{\mathbf{f}^{j}\right\}_{j \in J_{-0}}$ of prime aggregation functions such that each $\mathbf{f}^{j} \preceq \mathbf{f}$. Consider each $\bar{R} \in \operatorname{Im}(\mathbf{f})$. If $\bar{R} \neq \emptyset$ just consider the equivalence classes associated to each prime function according to Theorem 1: $\left\{\overline{\mathcal{S}}^{j}\right\}_{j \in J_{-0}} \subseteq$ $\widehat{\mathcal{S}} / \simeq_{\mathcal{D}\left(\mathcal{G}_{S}\right)}$. It is clear that for each prof $\mathcal{S}_{\mathcal{S}}$ such that $\mathcal{S} \in \cup_{j \in J_{-0}} \overline{\mathcal{S}}^{j}$ we have that there exists at least a $j \in J_{-0}$ that verifies that $\mathbf{f}^{j}\left(\right.$ prof $\left._{\mathcal{S}}\right)=\bar{R}$. On the other hand, if $R=\emptyset$ then consider $\overline{\mathcal{S}}^{0}$ the class of all the situations of the form $\left\langle S, R_{1}, \ldots, R_{n} ; \emptyset\right\rangle$. This class corresponds to the prime function $\mathbf{f}^{0}$ such that $\mathbf{f}^{0}\left(\operatorname{prof}_{\mathcal{S}}\right)=\emptyset$. Then, if we consider the class $J=J_{-0} \cup\{0\}$, we have that $\mathbf{f}\left(\right.$ prof $\left._{\mathcal{S}}\right)=\bar{R}$ for every $\mathcal{S} \in \cup_{j \in J} \overline{\mathcal{S}}^{j}$

Proof of Theorem 2 First of all, let us note that each chain $\mathcal{C}$ in $\left\langle\left\langle\mathbf{F}_{\mathcal{G}_{S}}, \preceq\right.\right.$ \rangle has a length, defined as its cardinality and denoted as $|\mathcal{C}|$. Consider first the case in which $|\mathcal{C}| \leq \aleph_{0}$, i.e. that $\mathcal{C}$ is countable. If $\mathcal{C}=\left\langle\mathbf{f}^{0}, \mathbf{f}^{1}, \ldots, \mathbf{f}^{|\mathcal{C}|}\right\rangle$, $\mathbf{f}^{0}$ is the only prime aggregation function in $\mathcal{C}$. According to Theorem 1, for $\mathbf{f}^{0}$ there exists a $\overline{\mathcal{S}}^{0} \in \widehat{\mathcal{S}} / \simeq_{\mathcal{D}\left(\mathcal{G}_{S}\right)}$ such that $\mathbf{f}^{0}(\operatorname{prof}(\mathcal{S}))=\bar{R}$ for every $\mathcal{S}=\left\langle S, R_{1}, \ldots, R_{n} ; \bar{R}\right\rangle \in \overline{\mathcal{S}}^{0}$. We will prove by induction that for each $\mathbf{f}^{k} \in \mathcal{C}$ there exists an element $\pi^{k}$ in a partition $\Pi^{\alpha^{k}}$ such that $\mathbf{f}^{k}(\operatorname{prof}(\mathcal{S}))=\bar{R}$ for every $\mathcal{S}=\left\langle S, R_{1}, \ldots, R_{n} ; \bar{R}\right\rangle \in \pi^{k}$. Moreover, that $\Pi^{\alpha^{k}}$ is a refinement of $\Pi^{\alpha^{k+1}}$ for each $k$ :

- Consider the case of $\mathbf{f}^{1}$. According to Proposition 2 we have that there exists $\left\{\overline{\mathcal{S}}^{j}\right\}_{j \in J} \subseteq \hat{\mathcal{S}} / \simeq_{\mathcal{D}\left(\mathcal{G}_{S}\right)}$ such that $\mathbf{f}\left(\right.$ prof $\left._{\mathcal{S}}\right)=\bar{R}$ for every $\mathcal{S}=$ $\left\langle S\right.$, prof $\left._{\mathcal{S}} ; \bar{R}\right\rangle \in \cup_{j \in J} \overline{\mathcal{S}}^{j}$. Suppose that $\overline{\mathcal{S}}^{0} \neq \overline{\mathcal{S}}^{j}$ for every $j \in J$, but then this means that $\mathbf{f}^{0}\left(\right.$ prof $\left._{\mathcal{S}}\right) \neq \mathbf{f}^{1}\left(\right.$ prof $\left._{\mathcal{S}}\right)$ for every $\mathcal{S} \in \overline{\mathcal{S}}^{0}$. Absurd, since we assumed that $\mathbf{f}^{0} \preceq \mathbf{f}^{1}$. Then $\overline{\mathcal{S}}^{0} \in\left\{\overline{\mathcal{S}}^{j}\right\}_{j \in J}$. That is, while $\mathbf{f}^{0}$ is supported by $\overline{\mathcal{S}}^{0} \in \widehat{\mathcal{S}} / \simeq_{\mathcal{D}\left(\mathcal{G}_{S}\right)}, \mathbf{f}^{1}$ is supported by $\overline{\mathcal{S}}^{0} \cup_{j \in J, j \neq 0} \overline{\mathcal{S}}^{j}$. If we call $\overline{\mathcal{S}}^{0}, \pi^{0}$ and $\widehat{\mathcal{S}} / \simeq_{\mathcal{D}\left(\mathcal{G}_{S}\right)}$, $\Pi^{\alpha_{0}}$, we see that if we denote $\overline{\mathcal{S}}^{0} \cup_{j \in J, j \neq 0} \overline{\mathcal{S}}^{j}$ as $\pi^{1}$, it is clear that $\pi_{0} \subseteq \pi_{1}$. On the other hand, $\pi_{1} \cup_{j^{\prime} \notin J} \overline{\mathcal{S}}^{j^{\prime}}=\widehat{\mathcal{S}}$ while $\pi_{1} \cap \overline{\mathcal{S}}^{\prime}=\emptyset$ for every $j^{\prime} \notin J$. That means that $\pi_{1}$ and $\left\{\overline{\mathcal{S}}^{\prime}\right\}_{j^{\prime} \notin J}$ constitute a partition of $\widehat{\mathcal{S}}$, which we call $\Pi^{\alpha_{1}}$. Since for $\pi^{0} \in \Pi^{\alpha^{0}}$, $\pi^{0} \subseteq \pi^{1}$ and for each $\overline{\mathcal{S}}^{j} \in \Pi^{\alpha^{0}}(j \in J), \overline{\mathcal{S}}^{j} \subseteq \pi^{1}$ while $\overline{\mathcal{S}}^{j} \in \Pi^{\alpha^{1}}$ for $j^{\prime} \notin J, \Pi^{\alpha^{1}}$ is a coarsening of $\Pi^{\alpha^{0}}$. 
- Assume that the result is valid up to $k$. That is, $\mathbf{f}^{k}$ is supported by a $\pi^{k} \in \Pi^{\alpha^{k}}$. Without loss of generality we assume that if $\mathbf{f}^{k}$ is supported, according to Proposition 2, by a family $\left\{\overline{\mathcal{S}}^{l}\right\}_{l \in L} \subseteq \widehat{\mathcal{S}} / \simeq_{\mathcal{D}\left(\mathcal{G}_{S}\right)}$ then $\pi^{k}=\cup_{l \in L} \overline{\mathcal{S}}^{l}$ and $\Pi^{\alpha^{k}}=\left\{\pi^{k},\left\{\overline{\mathcal{S}}^{l^{\prime}}\right\}_{l^{\prime} \notin L}\right\}$. Now consider the case of $\mathbf{f}^{k+1}$. Again, by Proposition 2, we have that there exists $\left\{\overline{\mathcal{S}}^{j}\right\}_{j \in J} \subseteq$ $\widehat{\mathcal{S}} / \simeq_{\mathcal{D}\left(\mathcal{G}_{n}, \mathcal{G}_{S}\right)}$ such that $\mathbf{f}^{k+1}\left(\right.$ prof $\left._{\mathcal{S}}\right)=\bar{R}$ for every $\mathcal{S} \in \cup_{j \in J} \overline{\mathcal{S}}^{j}$. Since $\mathbf{f}^{k} \preceq \mathbf{f}^{k+1}$ we have that $\mathbf{f}^{k}\left(\right.$ prof $\left._{\mathcal{S}}\right) \subseteq \mathbf{f}^{k+1}\left(\operatorname{prof}_{\mathcal{S}}\right)$ for every $\mathcal{S} \in \pi^{k}$. That is, $\pi^{k} \in\left\{\overline{\mathcal{S}}^{j}\right\}_{j \in J}$. Let us call the latter $\pi^{k+1}$. We have that $\pi^{k+1}$ and $\left\{\overline{\mathcal{S}}^{j^{\prime}}\right\}_{j^{\prime} \notin J}$ is a partition of $\widehat{\mathcal{S}}$ which we call $\Pi^{\alpha^{k+1}}$. It is clear that $\pi^{k} \subseteq \pi^{k+1}$ and $\overline{\mathcal{S}}^{j} \subseteq \pi^{k+1}$ for $j \in J$, while $\overline{\mathcal{S}}^{j} \in \Pi^{\alpha^{k+1}}$ for $j^{\prime} \notin J$. Therefore, $\Pi^{\alpha^{k+1}}$ is a coarsening of $\Pi^{\alpha^{k}}$.

Therefore, the claim is proved for every $k$.

Now consider the case in which $|\mathcal{C}|>\aleph_{0}$. Then, the set of indexes $k$ in $\mathcal{C}=\left\langle\mathbf{f}^{0}, \mathbf{f}^{1}, \ldots, \mathbf{f}^{|\mathcal{C}|}\right\rangle$ can be decomposed in two classes, those of the functions with either successor or limit indexes. That is, those indexes $k^{\prime}$ that have the form $k^{\prime}=k+1$ (the successor indexes) and those which verify that $k^{\prime}=\lim _{k<k^{\prime}} k$, the limit indexes. The proof for the countable length of $\mathcal{C}$ applies also for the successor indexes. The following is the proof for the limit indexes:

- Assume that the result is valid for each $k<k^{\prime}$ for a limit index $k^{\prime}$. That is, each $\mathbf{f}^{k}$ is supported by a $\pi^{k} \in \Pi^{\alpha^{k}}$ and, as $\mathbf{f}^{k}$ is supported by a family $\left\{\overline{\mathcal{S}}^{l}\right\}_{l \in L} \subseteq \widehat{\mathcal{S}} / \simeq_{\mathcal{D}\left(\mathcal{G}_{S}\right)}$ then $\pi^{k}=\cup_{l \in L} \overline{\mathcal{S}}^{l}$ and $\Pi^{\alpha^{k}}=\left\{\pi^{k},\left\{\overline{\mathcal{S}}^{\prime}\right\}_{l^{\prime} \notin L}\right\}$. Moreover, for each pair $k_{1}<k_{2}<k^{\prime}$, $\Pi^{\alpha^{k_{1}}}$ is a refinement of $\Pi^{\alpha^{k_{2}}}$. Let us now consider $\mathbf{f}^{k^{\prime}}$. By Proposition 2 we have that there exists $\left\{\overline{\mathcal{S}}^{j}\right\}_{j \in J} \subseteq \widehat{\mathcal{S}} / \simeq_{\mathcal{D}\left(\mathcal{G}_{S}\right)}$ such that $\mathbf{f}^{k^{\prime}}\left(\operatorname{prof}_{\mathcal{S}}\right)=\bar{R}$ for every $\mathcal{S} \in \cup_{j \in J} \overline{\mathcal{S}}^{j}$. For every $\mathbf{f}^{k} \preceq \mathbf{f}^{k+1}$ we have that $\mathbf{f}^{k}\left(\operatorname{prof}_{\mathcal{S}}\right) \subseteq \mathbf{f}^{k^{\prime}}\left(\right.$ prof $\left._{\mathcal{S}}\right)$ for every $\mathcal{S} \in \pi^{k}$. That is, each $\pi^{k} \in\left\{\overline{\mathcal{S}}^{j}\right\}_{j \in J}$. Let us call the latter $\pi^{k^{\prime}}$. We have that $\pi^{k^{\prime}}$ and $\left\{\overline{\mathcal{S}}^{j^{\prime}}\right\}_{j^{\prime} \notin J}$ is a partition of $\widehat{\mathcal{S}}$ which we call $\Pi^{\alpha^{k^{\prime}}}$. It is clear that for each $k<k^{\prime}, \pi^{k} \subseteq \pi^{k^{\prime}}$ and $\overline{\mathcal{S}}^{j} \subseteq \pi^{k^{\prime}}$ for $j \in J$, while $\overline{\mathcal{S}}^{j^{\prime}} \in \Pi^{\alpha^{k^{\prime}}}$ for $j^{\prime} \notin J$. Therefore, $\Pi^{\alpha^{k^{\prime}}}$ is a coarsening of $\left\{\Pi^{\alpha^{k}}\right\}_{k<k^{\prime}} \cdot \square$

Proof of Proposition 3 According to Theorem 2, $\mathcal{C}$ generates a chain of partitions, and particularly of equivalence classes, a $\pi^{k}$ for each $\mathbf{f}^{k} \in \mathcal{C}$. 
Since each $\pi^{k}$ is fully described by $\mathcal{D E C}\left(\mathbf{f}^{k}\right)$, we have that $\mathcal{C}$ generates also a sequence of the form $\mathcal{C}^{\mathcal{D E C}}=\left\{\mathcal{D E C}\left(\mathbf{f}^{k}\right)\right\}_{k=0}^{|\mathcal{C}|}$. To see that $\mathcal{C}^{\mathcal{D E C}}$ is a chain under $\subseteq$, just consider the fact that for $k<k^{\prime}$, for every profile prof $f_{\mathcal{S}}$ we have that $\mathbf{f}^{k}\left(\operatorname{prof}_{\mathcal{S}}\right) \subseteq \mathbf{f}^{k^{\prime}}\left(\operatorname{prof}_{\mathcal{S}}\right)$. Since, in particular $\mathbf{f}^{k}\left(\operatorname{prof}_{\mathcal{S}}\right)(s, t) \subseteq \mathbf{f}^{k^{\prime}}\left(\operatorname{prof}_{\mathcal{S}}\right)(s, t)$ for each situation $\mathcal{S}$ and every pair $s, t \in S$, the class of agents that are decisive over $s$ and $t$ for the former function, $D^{k}(\{s, t\}) \in \mathcal{F}_{\mathcal{G}_{S}}^{k}(\overline{\mathcal{S}}) \subseteq \mathcal{D E C}\left(\mathbf{f}^{k}\right)$ is also a class of decisive agents over $s$ and $t$ for the latter. That is, $D^{k}(\{s, t\}) \in \mathcal{F}_{\mathcal{G}_{S}}^{k^{\prime}}(\overline{\mathcal{S}}) \subseteq \mathcal{D} \mathcal{E C}\left(\mathbf{f}^{k^{\prime}}\right)$. In other words: $\mathcal{D E C}\left(\mathbf{f}^{k}\right) \subseteq \mathcal{D E C}\left(\mathbf{f}^{k^{\prime}}\right)$

Proof of Proposition 4 Trivial. Suppose $\mathcal{S}^{*} \in \mathcal{S U P \mathcal { P }}\left(\mathbf{f}^{j}\right)$ for $j<j^{*}$. Then, there would exist $\mathcal{S}^{\prime} \in \mathcal{S U P \mathcal { P }}\left(\mathbf{f}^{j^{*}}\right)$, such that for a given pair $s, t \in S$ and a permutation $\gamma, D^{\mathcal{S}^{*}}(\{s, t\}) \subseteq D^{\mathcal{S}^{\prime}}(\{\gamma(s), \gamma(t)\})$. Then, either $D^{\mathcal{S}^{*}}(\{s, t\})=D^{\mathcal{S}^{\prime}}(\{\gamma(s), \gamma(t)\})$ (in which case $\mathcal{S}^{*}$ is decision-equivalent to $\mathcal{S}^{\prime}$ and therefore $\left.\mathcal{S}^{*} \in \mathcal{S U P P}\left(\mathbf{f}^{j^{*}}\right)\right)$ or $D^{\mathcal{S}^{*}}(\{s, t\}) \subset D^{\mathcal{S}^{\prime}}(\{\gamma(s), \gamma(t)\})$. But then, since $\mathcal{D E C}\left(\mathbf{f}^{j}\right) \subseteq \mathcal{D E C}\left(\mathbf{f}^{j^{*}}\right)$, there will exist a decisive set for a pair $s$ and $t, D(\{s, t\}) \in \mathcal{D E C}\left(\mathbf{f}^{j^{*}}\right)$ for which there does not exist a of permutation $\gamma$ such that $D^{\mathcal{S}^{*}}(\{s, t\}) \subseteq D(\{\gamma(s), \gamma(t)\})$. Contradiction.

Proof of Proposition 7 By construction, each $\theta \in \cup \mathcal{H}(\mathbf{f})$ is $\theta=$ $\left\langle i, \mathcal{P}_{\mathcal{S}}, \mathbf{G}_{\mathbf{n}}, \mathbf{G}_{\mathbf{S}}\right\rangle$, i.e. a single individual and a pair of states over which she is decisive(plus the permutations that preserve her decisiveness). Then $\theta \in \mathbf{H}$ for a $\mathbf{H} \in \mathcal{H}(\mathbf{f})$. Define then $\Upsilon(\theta)$ as the corresponding $\mathbf{H}$.

Proof of Lemma 1 The first claim follows immediately from Definition 10 and Proposition 6. The characterization of the sentences in $\mathcal{A}^{\mathbf{f}}$ follows from the fact that sentences of the type $\phi_{\mathbf{H}}^{\forall}, \phi_{\mathbf{H}}^{\forall}, \phi_{\mathbf{H}}^{\exists}, \phi_{\mathbf{H}}^{\exists}$ or $\phi^{\triangle}$ can verified in $\mathcal{H}^{\mathbf{f}}$ independently of $S$ and $n$ being finite or not. 